\title{
Covariant phase space for gravity with boundaries: Metric versus tetrad formulations
}

\author{
J. Fernando Barbero G.๑, ${ }^{3,4, *}$ Juan Margalef-Bentabol ${ }^{1,3, \dagger}$ Valle Varo $\oplus^{2,3, \sharp}$ and Eduardo J. S. Villaseñor $\circledast^{2,3, \S}$ \\ ${ }^{1}$ Institute for Gravitation and the Cosmos and Physics Department, \\ Penn State University, Pennsylvania 16802, USA \\ ${ }^{2}$ Departamento de Matemáticas, Universidad Carlos III de Madrid, \\ Avda. de la Universidad 30, 28911 Leganés, Spain \\ ${ }^{3}$ Grupo de Teorías de Campos y Física Estadística, Instituto Gregorio Millán (UC3M), \\ Unidad Asociada al Instituto de Estructura de la Materia, CSIC, 28006 Madrid, Spain \\ ${ }^{4}$ Instituto de Estructura de la Materia, CSIC, Serrano 123, 28006 Madrid, Spain
}

(Received 5 May 2021; accepted 1 July 2021; published 18 August 2021)

\begin{abstract}
We use covariant phase space methods to study the metric and tetrad formulations of general relativity in a manifold with boundary and compare the results obtained in both approaches. Proving their equivalence has been a long-lasting problem that we solve here by using the cohomological approach provided by the relative bicomplex framework. This setting provides a clean and ambiguity-free way to describe the solution spaces and associated symplectic structures. We also compute several relevant charges in both schemes and show that they are equivalent, as expected.
\end{abstract}

DOI: 10.1103/PhysRevD.104.044048

\section{INTRODUCTION}

Space-time boundaries play a prominent role in classical and quantum general relativity (GR). Their applications range from black hole thermodynamics $[1,2]$ to the study of radiative modes at scri [3-6]. They are also fundamental to describe isolated and dynamical horizons [7-11] or in the definition of physical charges [12-15]. Boundary terms also control the dynamical properties of some midisuperspace models with "conical singularities" at spatial infinity such as Einstein-Rosen waves coupled to massless scalar fields [16-19].

Classical GR defined in a space-time without boundary may be alternatively described by the first order HilbertPalatini (or Holst) action in terms of tetrads or the EinsteinHilbert Lagrangian in metric variables. In particular, the corresponding Hamiltonian descriptions are equivalent. Moreover, by employing standard covariant phase space (CPS) methods, it is possible to see that their respective symplectic potentials differ by an exact form. Thus, when this form is integrated over a Cauchy slice $\Sigma$, the result vanishes as a consequence of Stokes's theorem. This shows that the presymplectic forms corresponding to the metric and the tetrad formalisms are equivalent in the space of solutions.

\footnotetext{
*fbarbero@iem.cfmac.csic.es

juanmargalef@psu.edu

valle@cvb.es

§ejsanche@math.uc3m.es
}

The situation changes substantially when boundaries (possible at conformal infinity) are present. Some of the concerns regarding the equivalence of the tetrad and metric formulations may be attributed to the choice of bulk and boundary Lagrangians. In fact, many discrepancies found and discussed in the literature [20-23] are to be expected since the boundary dynamics is not always explicit and the solution spaces are not fully specified. To avoid these problems, it is crucial to choose variational principles such that the equivalence of the dynamics they describe, both in the bulk and at the boundaries, is guaranteed. The choice of the bulk and boundary Langrangians will determine the presymplectic structure in the solution space as well as the Noether charges.

The purpose of this paper is to prove that the metric and tetrad formulations are equivalent in the CPS framework with Dirichlet boundary conditions (BC) and (homogeneous) Neumann BC (the same methods can be applied to other BC). This proof has profound physical implications. Although at first sight this may appear as a purely mathematical result, nonequivalence would make physical quantities formulation dependent. For instance, the conserved charges might differ at conformal infinity and/or at the black hole horizon. As suggested in [20], this would have repercussions in the thermodynamics of black holes.

To prove the equivalence, we will rely on the relative bicomplex framework, a formalism developed in [15] which is cohomological in nature so no ad hoc choices are required. This formalism will allow us to establish the equality of the metric and tetrad symplectic potentials in the 
relative cohomology (equality up to a relative exact form as explained in the Appendix). Consequently, the respective presymplectic forms in the solution space are equivalent and, as expected, there is a precise correspondence between the Noether charges in both formulations. As a direct result of our discussion, we will consider the asymptotically flat case and rederive the well-known formula for the ArnowittDeser-Misner (ADM) energy.

The paper is structured as follows. In the next section, we present some of the geometric concepts relevant for the implementation of the CPS algorithm developed in [15]. In Sec. III, we apply this algorithm to the metric formulation of GR. Section IV presents an analogous study for the tetrad (or vielbein) formalism. In Sec. V both formulations are compared and shown to be equivalent. We present our conclusions in the last section of the paper. We have also included in the Appendix a short summary of the relative bicomplex framework together with some computational details. Throughout the paper, we will use a mixed notation combining explicit indices and index-free expressions. The idea is to use each of them whenever they are useful: the former is more standard and sometimes more explicit, while the latter simplifies significantly some of the computations. For the benefit of the reader, relevant results are presented in both notations.

\section{THE GEOMETRIC ARENA}

\section{A. $M$ as a space-time}

Let $M$ be a connected and oriented $n$-manifold admitting a foliation by Cauchy hypersurfaces. Without loss of generality, $M=I \times \Sigma$ for some interval $I=\left[t_{i}, t_{f}\right]$, $\left(t_{i}<t_{f}\right)$ and some $(n-1)$-manifold $\Sigma$ with boundary $\partial \Sigma$ (possibly empty). Denoting $\Sigma_{i}=\left\{t_{i}\right\} \times \Sigma$ and $\Sigma_{f}=\left\{t_{f}\right\} \times \Sigma$, we split $\partial M$ into three distinguished parts

$$
\partial M=\Sigma_{i} \cup \partial_{L} M \cup \Sigma_{f}
$$

where $\Sigma_{i, f}$ are the "lids" and $\partial_{L} M:=I \times \partial \Sigma$ the "lateral boundary." $M=I \times \Sigma$ is a manifold with corners $\partial \Sigma_{i} \cup \partial \Sigma_{f}$, which, as a set are $\partial\left(\partial_{L} M\right)$. The following diagram summarizes relevant notational information about embeddings and the induced geometric objects:

$$
\begin{gathered}
(\Sigma, \gamma, D,\{a, b, \ldots\}) \underset{\left(\imath, n^{\alpha}\right)}{\stackrel{(}{\longrightarrow}\left(\bar{\jmath}, \mu^{a}\right) \uparrow}(M, \nabla,\{\alpha, \beta, \ldots\}) \\
(\partial \Sigma, \bar{\gamma}, \bar{D},\{\bar{a}, \bar{b}, \ldots\}) \underset{\left(\bar{\imath}, \nu^{\alpha}\right)}{\longrightarrow}(\partial M, \bar{g}, \bar{\nabla},\{\bar{\alpha}, \bar{\beta}, \ldots\})
\end{gathered}
$$

The entries in each 4-tuple in (2.1) are the manifold, the (nondegenerate) metric or pulled-back metric, its associated Levi-Civita connection, and the abstract indices used to describe tensors in the manifold. The arrow labels specify the notation used for embeddings and normal unit vector fields. Horizontal arrows are associated with future pointing normal unit vector fields and vertical arrows are used for outward pointing normal unit vector fields at the boundary. The notation just introduced is not consistent for the bottom lid $\Sigma_{i}$, as it may be thought of as a spacelike hypersurface embedded by $l_{i}$ or considered as part of $\partial M$. $\Sigma_{i}$ often appears as the boundary of $M$, so we choose the outwards (past pointing) convention in this case. An overline is often used to denote objects that live exclusively at the boundary, such as $\bar{g}$, which in index notation reads $\bar{g}_{\bar{\alpha} \bar{\beta}}$. Notice that the embeddings $J$ and $\bar{J}$ are fixed since the boundaries are also fixed. However, $\iota$ and $\bar{l}$, which embed $(\Sigma, \partial \Sigma)$ into $(M, \partial M)$, can be chosen among the Cauchy embeddings satisfying $l(\partial \Sigma) \subset \partial_{L} M$.

As $(M, g)$ is oriented, we have the metric volume form $\operatorname{vol}_{g}$ that assigns the value 1 to every positive orthonormal basis. We orient $\Sigma$ and $\partial_{L} M$ with $\operatorname{vol}_{\gamma}$ and $\operatorname{vol}_{\bar{g}}$, respectively given by

$l^{*}\left(l_{\vec{U}} \operatorname{Vol}_{g}\right)=-n_{\alpha} U^{\alpha} \operatorname{vol}_{\gamma}, \quad J^{*}\left(l_{\vec{U}} \operatorname{vol}_{g}\right)=\nu_{\alpha} U^{\alpha} \operatorname{vol}_{\bar{g}}$

for every vector field $\vec{U}$. These orientations are the ones for which Stokes's theorem holds in its usual form. Finally, $\partial \Sigma$ can be oriented as the boundary of $\Sigma$. Thus $\operatorname{vol}_{\bar{\gamma}}$ is given by

$\bar{J}^{*}\left(l_{\vec{V}} \operatorname{Vol}_{\gamma}\right)=\mu_{a} V^{a} \operatorname{vol}_{\bar{\gamma}} \rightarrow \bar{l}^{*}\left(l_{\vec{W}} \operatorname{Vol}_{\bar{g}}\right)=+\bar{m}_{\bar{\alpha}} W^{\bar{\alpha}} \operatorname{vol}_{\bar{\gamma}}$.

Notice that if we use Stokes's theorem from $\partial_{L} M$ to $\partial\left(\partial_{L} M\right)=\partial \Sigma_{i} \cup \partial \Sigma_{f}$, a minus sign appears in the integral over $\partial \Sigma_{i}$.

\section{B. From $M$-vector fields to $\mathcal{F}$-vector fields}

Let $\mathcal{F}$ be a space of tensor fields on $M$ (sections of a certain bundle $E \stackrel{\pi}{\rightarrow} M$ ) that may be thought of as an infinite dimensional differential manifold where we assume that the usual differential objects-like tensor fields, the exterior derivative $\mathbb{d}$, the interior derivative $l$, the wedge $\mathbb{A}$, or the Lie derivative $\mathcal{L}$-are well defined (see the appendix of [15] for more details about this and other technicalities). As a reminder for the reader, most objects defined over the space of fields are denoted with a double font. Given a vector field $\xi^{\alpha}$ on $M$, let us associate to it a canonical vector field $\mathbb{X}_{\xi}$ on $\mathcal{F}$. To that purpose, we assume that $\xi$ is tangent to $\partial_{L} M$ but not necessarily to $\Sigma_{i}$ and $\Sigma_{f}$ (notice that we can always extend the interval $\left[t_{i}, t_{f}\right]$ to avoid problems at the lids) and take advantage of the fact that a field $\phi^{r}$ ( $r$ ranging over the number of fields of the theory) can be interpreted in two ways:

(i) As a tensor field on $M$. That is, a section $\phi^{r}: M \rightarrow$ $E^{r}$ of some bundle $E^{r} \stackrel{\pi}{\rightarrow} M$ such that $\phi_{p}^{r}:=\phi^{r}(p) \in E_{p}^{r}:=\pi^{-1}(p)$. In particular, we can take its Lie derivative $\left(\mathcal{L}_{\xi} \phi^{r}\right)_{p}=\left.\partial_{\tau}\right|_{0}\left(\varphi_{\tau}^{\xi}\right)^{*} \phi_{p}^{r}$, 
where $\left\{\varphi_{\tau}^{\xi}\right\}_{\tau} \subset \operatorname{Diff}(M)$ is the flow of $\xi$. If $\mathcal{F}$ is reasonable enough, as is the case in this paper, we will have $\mathcal{L}_{\xi} \phi \in \mathcal{F}$.

(ii) As a point of $\mathcal{F}$. A vector field $\mathbb{X}_{\xi}$ of $\mathcal{F}$ is a section of the tangent bundle $T \mathcal{F}$ i.e., $\left(\mathbb{X}_{\xi}\right)_{\phi}:=\mathbb{X}_{\xi}(\phi) \in$ $T_{\phi} \mathcal{F} \cong \mathcal{F}$. The last isomorphism comes from the fact that the field space $\mathcal{F}$ is linear in this case. The nonlinear case is not as straightforward, but it is possible to carry out similar constructions in concrete examples.

Taking into account these remarks, we define

$$
\left(\mathbb{X}_{\xi}^{r}\right)_{\phi}=\mathcal{L}_{\xi} \phi^{r} \equiv \mathcal{L}_{\mathbb{X}_{\xi}} \phi^{r}=\mathcal{L}_{\xi} \phi^{r} .
$$

Notice that $\mathbb{d} \phi^{r}$ (which appears in the definition of the Lie derivative $\mathcal{L}$ through Cartan's magic formula) has to be interpreted as the exterior derivative of the evaluation function.

\section{CPS algorithm}

This paper relies heavily on the results of [15]. For convenience, a summary has been included in the Appendix, but we list here the main steps of the CPS algorithm since it will be used throughout the paper. This algorithm provides an ambiguity-free method to construct a presymplectic structure in the space of solutions canonically associated with the action of the theory.

(0) Given the action $\mathbb{S}: \mathcal{F} \rightarrow \mathbb{R}$ describing the dynamics of a particular field theory, choose any Lagrangian pair $(L, \bar{\ell})$ such that

$$
\mathbb{S}=\int_{M} L-\int_{\partial M} \bar{\ell}
$$

(1) Compute $\mathbb{d} L=E_{r} \wedge \mathbb{d} \phi^{r}+\mathrm{d} \Theta$ and choose any $\Theta$ compatible with the previous expression (uniquely defined up to a d-exact form).

(2) Compute $\mathbb{d} \bar{\ell}-J^{*} \Theta=\bar{b}_{r} \wedge \mathbb{d} \phi^{r}-\mathrm{d} \bar{\theta}$ over $\partial_{L} M$ and choose any admissible $\bar{\theta}$.

(3) Define $\operatorname{Sol}(\mathbb{S})=\left\{\phi \in \mathcal{F} \mid E_{r}(\phi)=0, \bar{b}_{r}(\phi)=0\right\}$ and the inclusion $J_{\mathbb{S}}: \operatorname{Sol}(\mathbb{S}) \hookrightarrow \mathcal{F}$.
(4) Compute the presymplectic structure canonically associated with $\mathbb{S}$

$$
\mathbb{\Omega}_{\mathbb{S}}^{l}:=\mathbb{d}\left(\int_{\Sigma} l^{*} \Theta-\int_{\partial \Sigma} i^{*} \bar{\theta}\right) \rightarrow \mathbb{\Omega}_{\mathbb{S}}=J_{\mathbb{S}}^{*} \mathbb{\Omega}_{\mathbb{S}}^{l}
$$

where $l: \Sigma \hookrightarrow M$ is any Cauchy embedding and $\bar{l}:=$ $\left.\imath\right|_{\partial \Sigma}: \partial \Sigma \hookrightarrow \partial_{L} M$ its restriction. $\mathbb{\Omega}_{\mathbb{S}}$ does not depend on $l$.

It is important to notice that it is not always possible to perform the second step. If $\bar{b}_{r}$ and $\bar{\theta}$ do not exist (their existence does not depend on the chosen $\Theta$ ), that means that the theory is not well defined and hence we will have to change $\mathcal{F}$ and/or the action [hence $(L, \bar{\ell})]$ to ensure that the equality holds. Once we have the presymplectic structure, the following two steps provide important additional information about the theory at hand.

(5) Study symmetries; i.e., find out if $\mathbb{X}_{\xi}$ is a d-symmetry and obtain the $\xi$ currents and $\xi$ charges:

$$
\begin{aligned}
& J_{\xi}:=l_{\xi} L-l_{\chi_{\xi}} \Theta, \\
& \bar{J}_{\xi}:=-l_{\xi} \bar{\ell}-l_{\Upsilon_{\xi}} \bar{\theta}, \\
& \mathbb{Q}_{\xi}^{l}:=\int_{\Sigma} \imath^{*} J_{\xi}-\int_{\partial \Sigma} \bar{l}^{*} \bar{J}_{\xi} .
\end{aligned}
$$

(6) Compare $\mathbb{\Omega}_{\mathbb{S}}$ with the presymplectic structure obtained in the standard Hamiltonian formulation.

\section{GENERAL RELATIVITY IN TERMS OF METRICS}

\section{A. Step 0: Action}

Let us consider the following spaces of metrics on $M$ :

$$
\mathcal{F}_{N}^{(m)}=\operatorname{Met}(M), \quad \mathcal{F}_{D}^{(m)}=\left\{g \in \operatorname{Met}(M) \mid \bar{g}:=J^{*} g \text { fixed }\right\}
$$

that we will refer to as Neumann and Dirichlet metrics respectively. Here $\operatorname{Met}(M)$ is the space of Lorentzian metrics on $M$ such that the lateral boundary is timelike and the lids are spacelike (it is an open set of the space of all Lorentzian metrics on $M$ ). Consider also the actions $\mathbb{S}_{N}^{(m)}$ and $\mathbb{S}_{D}^{(m)}$ both equal to

$$
\mathbb{S}_{\mathrm{GR}}^{(m)}(g)=\int_{M} L_{\mathrm{EH}}^{(m)}(g)-\int_{\partial_{L} M} \bar{\ell}_{\mathrm{GHY}}^{(m)}(g), \quad \begin{aligned}
& L_{\mathrm{EH}}^{(m)}(g)=\left(R_{g}-2 \Lambda\right) \mathrm{vol}_{g}, \\
& \bar{\ell}_{\mathrm{GHY}}^{(m)}(g)=-2 \operatorname{Tr}_{\bar{g}}(\bar{K}) \operatorname{vol}_{\bar{g}}
\end{aligned}
$$

but suitably restricted to these spaces of metrics. Our Lagrangian pair $\left(L_{\mathrm{EH}}^{(m)}, \bar{\ell}_{\mathrm{GHY}}^{(m)}\right)$ consists of the EinsteinHilbert term with cosmological constant in the bulk and the Gibbons-Hawking-York term on the lateral boundary. $R_{g}$ denotes the $g$-scalar curvature (following the signs conventions of [24]), $\operatorname{vol}_{g}$ and $\operatorname{vol}_{\bar{g}}$ the metric volume forms, and $\bar{K}$ the extrinsic curvature of $\partial_{L} M \subset M$ given by

$$
\bar{K}:=\frac{1}{2} J^{*} \mathcal{L}_{\vec{\nu}} g \equiv \bar{K}_{\bar{\alpha} \bar{\beta}}=J_{\bar{\alpha}}^{\alpha} J_{\bar{\beta}}^{\beta} \nabla_{\alpha} \nu_{\beta}
$$

where $\vec{\nu}$ is the $g$-normal vector field to $\partial_{L} M$ with $\nu_{\alpha} \nu^{\alpha}=+1$. 


\section{B. Step 1 and 2: Variations}

According to the computations given in the Appendix, we have

$$
\begin{array}{r}
\mathbb{d} L_{\mathrm{EH}}^{(m)}=E_{(m)}^{\alpha \beta} \wedge \mathbb{d} g_{\alpha \beta}+\mathrm{d} \Theta_{(m)}, \\
\mathbb{d} \bar{\ell}_{\mathrm{GHY}}^{(m)}-J^{*} \Theta_{(m)}=\bar{b}_{(m)}^{\bar{\alpha} \bar{\beta}} \wedge \mathbb{d} \bar{g}_{\bar{\alpha} \bar{\beta}}-\mathrm{d} \bar{\theta}_{(m)}
\end{array}
$$

where

$$
\begin{aligned}
E_{(m)}^{\alpha \beta}(g) & =-\left(\operatorname{Ric}^{\alpha \beta}-\left(\frac{R_{g}}{2}-\Lambda\right) g^{\alpha \beta}\right) \operatorname{vol}_{g}, \\
\bar{b}_{(m)}^{\bar{\alpha} \bar{\beta}}(g) & =-\left(\bar{K}^{\bar{\alpha} \bar{\beta}}-\bar{K} \bar{g}^{\bar{\alpha} \bar{\beta}}\right) \operatorname{vol}_{\bar{g}}, \\
\Theta_{(m)}(g) & =l_{\vec{W}} \operatorname{Vol}_{g}, \quad \bar{\theta}_{(m)}(g)=l_{\vec{V}} \operatorname{Vol}_{\bar{g}}, \\
W_{\alpha} & =\nabla^{\beta} \mathbb{d} g_{\alpha \beta}-g^{\beta \gamma} \nabla_{\alpha} \mathbb{d} g_{\beta \gamma}, \quad \bar{V}_{\bar{\alpha}}=-J_{\bar{\alpha}}^{\alpha} \nu^{\beta} \mathbb{d} g_{\alpha \beta} .
\end{aligned}
$$

\section{Step 3: Space of solutions}

Once we know the basic variations, we can compute the variation of the action

$\mathbb{d}_{g} \mathbb{S} \stackrel{\mathcal{L}}{=} \int_{M} E_{(m)}^{\alpha \beta}(g) \mathbb{M} \mathbb{d} g_{\alpha \beta}-\int_{\partial_{L} M} \bar{b}_{(m)}^{\bar{\alpha} \bar{\beta}}(g) \mathbb{A} \mathbb{d} \bar{g}_{\bar{\alpha} \bar{\beta}}$

where $\underline{\mathcal{L}}$ denotes equality up to integrals over the lids. Notice that those integrals are unimportant because the values of the dynamical fields on the lids are kept fixed when obtaining the equations of motion of the theory. The solutions obtained from $\mathbb{S}_{N}^{(m)}$ are given by metrics $g \in \mathcal{F}_{N}^{(m)}$ satisfying Einstein's equations $E_{(m)}(g)=0$ and the Neumann boundary condition $\bar{b}_{(m)}(g)=0$, whereas those from $\mathbb{S}_{D}^{(m)}$ correspond to metrics $g \in \mathcal{F}_{D}^{(m)}$ satisfying Einstein's equation and no additional BC. Indeed, in the latter case the boundary integral of (3.1) vanishes as a consequence of the Dirichlet BC introduced a priori on the definition of $\mathcal{F}_{D}^{(m)}$.

Remark.-It is important to realize that a boundary term must be included in the two theories that we consider (i.e., when no a priori $\mathrm{BC}$ are imposed on the space of fields and with Dirichlet BC). Otherwise, as explained in Sec. II C, the second step cannot be performed and thus the space of solutions is not well defined. Nonetheless, it is possible to impose homogeneous Neumann BC a priori and include a different boundary term [25] (which vanishes for dimension 4). However, as we have shown, this is not necessary. Finally, it is worth noting that nontrivial BC may lead to constrained variations, which must be properly handled.

\section{Step 4: Symplectic form}

Given a Cauchy embedding $l: \Sigma \hookrightarrow M$, we compute the presymplectic structure canonically associated with the action as

$$
\mathbb{\Omega}_{(m)}^{l}=\mathbb{d}\left(\int_{\Sigma} l^{*} \Theta_{(m)}-\int_{\partial \Sigma} \bar{l}^{*} \bar{\theta}_{(m)}\right) .
$$

Using the results of the previous sections and the computations of the Appendix, we obtain:

$$
\begin{aligned}
& \mathbb{\Omega}_{(m)}^{l}=\frac{1}{2} \int_{\Sigma} n^{\alpha} \delta_{\alpha \beta \zeta}^{\lambda \eta \sigma} g^{\beta \rho} g^{\zeta \phi} \mathbb{d} g_{\eta \rho} \mathbb{\mathbb { }} \nabla_{\lambda} \llbracket g_{\sigma \phi} \operatorname{vol}_{\gamma} \\
& -\frac{1}{2} \int_{\partial \Sigma}\left(n^{\alpha} g^{\beta \eta} \mu^{\lambda}+m^{\alpha} \nu^{\beta} \bar{g}^{\bar{\eta}} \bar{\lambda} J_{\bar{\eta}}^{\eta} J_{\bar{\lambda}}^{\lambda}\right) \mathbb{d} g_{\alpha \beta} \mathbb{\mathbb { N }} \mathbb{d} g_{\eta \lambda} \operatorname{vol}_{\bar{\gamma}} \text {. }
\end{aligned}
$$

As indicated in (2.1), $n^{\alpha}$ is the $g$-normal to $t(\Sigma) \subset M, \nu^{\beta}$ is the $g$-normal to $\partial_{L} M \subset M, m^{\alpha}$ is the pushforward of the $\bar{g}$ normal to $\bar{l}(\partial \Sigma) \subset \partial_{L} M$, and $\mu^{\lambda}$ is the pushforward of the $\gamma-$ normal to $\partial \Sigma \subset \Sigma$. We have also used the generalized Kronecker delta

$$
\delta_{\alpha_{1} \cdots \alpha_{s}}^{\beta_{1} \cdots \beta_{s}}=\left|\begin{array}{ccc}
\delta_{\alpha_{1}}^{\beta_{1}} & \cdots & \delta_{\alpha_{1}}^{\beta_{s}} \\
\vdots & & \vdots \\
\delta_{\alpha_{s}}^{\beta_{1}} & \cdots & \delta_{\alpha_{s}}^{\beta_{s}}
\end{array}\right| .
$$

\section{E. Step 5: Charges}

The Noether currents associated with an arbitrary vector field $\xi^{\alpha}$ tangent to the lateral boundary are computed in the Appendix. The result is

$$
\begin{aligned}
& J_{\xi}^{(m)}=\mathrm{d} Q_{\xi}^{(m)}+2 \star_{g}\left(l_{\xi} \tilde{E}_{(m)}\right), \\
& \bar{J}_{\xi}^{(m)}=J^{*} Q_{\xi}^{(m)}-2 \star_{\bar{g}}\left(l_{\xi} \tilde{b}_{(m)}\right),
\end{aligned}
$$

where $Q_{\xi}^{(m)}=\star_{g} \mathrm{~d} \xi$ is the $\xi$-Noether potential and $\left(\tilde{E}_{(m)}, \tilde{b}_{(m)}\right)$ are the 2-tensors that multiply the top forms in $\left(E_{(m)}, \bar{b}_{(m)}\right)$. As usual, $\star_{g}$ is the $g$-Hodge dual operator and, in order to ease the notation, we represent also by $\xi$ the 1 -form metrically equivalent to the vector field $\xi$. The $\xi$ charge is then given by

$$
\mathbb{Q}_{\xi,(m)}^{l}=2 \int_{\Sigma} l^{*} \star_{g}\left(l_{\xi} \tilde{E}_{(m)}\right)+2 \int_{\partial \Sigma} \bar{l}^{*} \star_{\bar{g}}\left(l_{\bar{\xi}} \tilde{b}_{(m)}\right) .
$$

The last expression, when pulled back to $\operatorname{Sol}\left(\mathbb{S}_{N}^{(m)}\right) \subset \mathcal{F}_{N}^{(m)}$, vanishes as expected because the theory is diffeomorphism invariant [15]. However, over $\operatorname{Sol}\left(\mathbb{S}_{D}^{(m)}\right) \subset \mathcal{F}_{D}^{(m)}$, the boundary integral is not necessarily zero as $\bar{b}_{(m)}$ does not vanish in general. Notice that fixing the metric on the boundary breaks diffeomorphism invariance. 


\section{F. Step 6: Comparing with the Hamiltonian formulation}

In the case $\partial \Sigma=\varnothing$, the equivalence between the CPS presymplectic form obtained in step 4 and the canonical ADM presymplectic form has been established in [26,27]. The case with boundaries is not as straightforward and will be studied elsewhere.

\section{G. The asymptotically flat case}

As a further application of the CPS algorithm, let us obtain the ADM energy of an asymptotically flat spacetime (see $[28,29]$ for similar approaches). For that purpose, consider $M=\Sigma \times \mathbb{R}$ endowed with the Minkowski metric $g_{0}=-\mathrm{d} t^{2}+\sigma$. Then $\Sigma$ is a 3 -manifold with Euclidean metric $\sigma$ and boundary $\partial \Sigma=\mathbb{S}_{r}^{2}$, a 2-sphere of $\sigma$ radius $r_{0}$. Take a small perturbation $h \in T_{g_{0}} \operatorname{Met}(M)$, such that $\bar{h}:=$ $J^{*} h=0$ (compatible with the Dirichlet condition, otherwise the charge is zero as the theory is diffeomorphism invariant). The $\xi$ charge given by (3.4) restricted to the space of solutions is given by

$$
\mathbb{Q}_{\xi}=2 \int_{\partial \Sigma} \bar{\xi}_{\bar{\alpha}} \bar{m}_{\bar{\beta}}\left(\bar{K}^{\bar{\alpha} \bar{\beta}}-\bar{K} \bar{g}^{\bar{\alpha} \bar{\beta}}\right) \operatorname{vol}_{\bar{\gamma}} .
$$

Expanding the integrand in terms of $h$, and labeling with a circle the quantities associated with $g_{0}$ (the "background" objects), we obtain

$$
\begin{aligned}
\mathbb{Q}_{\xi}= & 2 \int_{\partial \Sigma} \bar{\xi}_{\bar{\alpha}}\left(\stackrel{\circ}{\bar{m}}_{\bar{\beta}}+\mathcal{L}_{h} \bar{m}_{\bar{\beta}}\right)\left(\stackrel{\circ}{\bar{K}}^{\bar{\alpha} \bar{\beta}}+\mathcal{L}_{h} \bar{K}^{\bar{\alpha} \bar{\beta}}\right. \\
& \left.-\left(\stackrel{\circ}{\bar{K}}+\mathcal{L}_{h} \bar{K}\right)\left(\stackrel{\circ}{\bar{g}}^{\bar{\alpha} \bar{\beta}}-\mathcal{L}_{h} \bar{g}^{\bar{\alpha} \bar{\beta}}\right)\right)\left(\operatorname{vol}_{\bar{\sigma}}+\mathcal{L}_{h} \operatorname{vol}_{\gamma}\right)+\cdots
\end{aligned}
$$

where we omit higher order terms in $h$ as they vanish when $r_{0} \rightarrow \infty$ if we consider $\xi:=n$ and the inertial foliation $\{\{t\} \times \Sigma\}_{t}$ (in particular, we take $\vec{n}=\partial_{t}$ ).

Using the decomposition of the normal $\nu_{\alpha}=-\nu_{\perp} n_{\alpha}+$ $\nu_{\alpha}^{\top}$ and some of the results of Sec. A 2, we have

$$
\begin{aligned}
\mathcal{L}_{h} \bar{m}_{\bar{\alpha}} & =J_{\bar{\alpha}}^{\alpha} \mathcal{L}_{h}\left(\frac{n_{\alpha}+\nu_{\perp} \nu_{\alpha}}{\left|\nu^{\top}\right|}\right) \\
& =l_{h}\left(\frac{1}{\left|\nu^{\top}\right|} J_{\bar{\alpha}}^{\alpha} \mathbb{d} n_{\alpha}-\bar{m}_{\bar{\alpha}} \frac{\nu_{\perp}}{\left|\nu^{\top}\right|} \mathbb{d} \nu_{\perp}\right) \\
& =\frac{1}{\left|\nu^{\top}\right|} J_{\bar{\alpha}}^{\alpha} l_{h}\left(\frac{-1}{2} n_{\alpha} n^{\beta} n^{\gamma} \llbracket g_{\beta \gamma}\right)-\bar{m}_{\bar{\alpha}} \frac{\stackrel{\nu}{\perp}_{\perp}}{\left|\nu^{\top}\right|} \mathcal{L}_{h} \nu_{\perp} \\
& =-\frac{1}{2} \bar{\xi}_{\bar{\alpha}} \xi^{\beta} \xi^{\gamma} h_{\beta \gamma}+0=-\frac{1}{2} \bar{\xi}_{\bar{\alpha}} \bar{\xi}^{\bar{\xi} \overline{\xi^{\gamma}} \bar{h}_{\bar{\beta} \bar{\gamma}}}=0
\end{aligned}
$$

where we have used that $\stackrel{\circ}{\perp}_{\perp}=0, \xi=n$ and the fact that $\xi$ is tangent to the lateral boundary (hence we can replace $h$ with $\bar{h}$, which is zero). Notice that the previous computation is performed at the point $g_{0}$ in the space of fields and at the lateral space-time boundary.
This computation together with $\bar{m}_{\bar{\beta}}=\bar{\xi}_{\bar{\beta}}$ takes care of the first parentheses of $\mathbb{Q}_{\xi}$. The last one can also be easily computed by using the variation of the volume given in Sec. A 2. Let us now deal with the term in the second parentheses. First notice that $\bar{K}^{\bar{\alpha} \bar{\beta}}$ vanishes when contracted with $\bar{\xi}_{\bar{\alpha}} \bar{\xi}_{\bar{\beta}}$ (in coordinates this would be $\bar{K}^{t t}$ ), while $\mathcal{L}_{h} \bar{g}^{\bar{\alpha}} \bar{\beta}=\bar{h}=0$. A long but straightforward computation gives the following variations:

$$
\begin{aligned}
\bar{\xi}_{\bar{\alpha}} \bar{\xi}_{\bar{\beta}} \mathcal{L}_{h} \bar{K}^{\bar{\alpha} \bar{\beta}}= & \frac{1}{2} \xi^{\alpha} \xi^{\beta}\left(\stackrel{\circ}{\nabla}_{\vec{\nu}} h_{\alpha \beta}-2 \nu^{\gamma} \stackrel{\circ}{\nabla}_{\alpha} h_{\beta \gamma}\right), \\
\mathcal{L}_{h} \bar{K}= & \frac{1}{2} l_{a}^{\alpha} l_{b}^{\beta} \gamma^{a b}\left(\stackrel{\circ}{\nabla}_{\vec{\nu}} h_{\alpha \beta}-\nu^{\gamma} \stackrel{\circ}{\nabla}_{\alpha} h_{\beta \gamma}\right) \\
& -\frac{1}{2} \xi^{\alpha} \xi \beta\left(\stackrel{\circ}{\nabla}_{\vec{\nu}} h_{\alpha \beta}-2 \nu^{\gamma} \stackrel{\circ}{\nabla}_{\alpha} h_{\beta \gamma}\right) \\
& -\frac{1}{2} \stackrel{\circ}{D}_{\bar{a}}\left(\bar{\gamma}^{\bar{a}} \bar{b} \bar{J}_{\bar{b}}^{\alpha} J_{\bar{\alpha}}^{\alpha}\left(l_{\vec{\nu}} h\right)_{\alpha}\right) \\
& +\frac{1}{2} \xi^{\alpha} \nu^{\beta} \mathcal{L}_{\vec{n}} g_{\alpha \beta} \xi^{\gamma \gamma} \nu^{\mu} h_{\gamma \mu} .
\end{aligned}
$$

As the result is independent of the Cauchy slice, we introduce coordinates $\left\{t, x_{1}, x_{2}, x_{3}\right\}$ and take, as we mentioned before, a Cauchy slice given by $\Sigma=\left\{t=t_{0}\right\}$, then

$$
\vec{\nu}=\frac{1}{r_{0}}\left(0, x_{1}, x_{2}, x_{3}\right) .
$$

Notice that everything is constant along the $t$ direction (which is the $\xi$ direction because $\vec{n}=\partial_{t}$ ). Thus we have

$\stackrel{\circ}{\bar{K}}_{\bar{\alpha} \bar{\beta}}=J_{\bar{\alpha}}^{\alpha} J_{\bar{\beta}}^{\beta} \stackrel{\circ}{\nabla}_{\alpha} \nu_{\beta}, \quad \stackrel{\circ}{\bar{K}}_{A B}=\frac{1}{r_{0}^{3}}\left(\delta_{A B} r_{0}^{2}-x_{A} x_{B}\right), \quad \stackrel{\circ}{\bar{K}}=\frac{D-2}{r_{0}}$

where here $A, B=1,2,3$ label the coordinates. Finally, notice that the integral of $h \bar{K} \operatorname{vol}_{\bar{\sigma}}$ goes to zero in the limit $r_{0} \rightarrow \infty$ and $l_{\xi} \bar{K}=0$ because the normal is constant along the foliation. Putting everything together, we obtain

$$
\mathbb{Q}_{\xi}=\int_{\partial \Sigma}\left(\stackrel{\circ}{\bar{K}}+\gamma^{B C} \nu^{A}\left(\stackrel{\circ}{\nabla}_{A} h_{B C}-\stackrel{\circ}{\nabla}_{B} h_{C A}\right)\right) \operatorname{vol}_{\bar{\sigma}}+\cdots .
$$

A final comment is now in order. As we can see we have a divergent constant term in the limit $r_{0} \rightarrow \infty$. To remove it, we introduce the following $r_{0}$-dependent boundary Lagrangian:

$$
\bar{\ell}_{\left(r_{0}\right)}=2\left(\operatorname{Tr}_{\bar{g}}(\stackrel{\circ}{\bar{K}})-\operatorname{Tr}_{\bar{g}}(\bar{K})\right) \operatorname{vol}_{\bar{g}} .
$$

By doing this, the constant term does not appear, the limit is well defined, and we recover the well-known expression for the ADM energy. 


\section{GENERAL RELATIVITY IN TERMS OF TETRADS}

\section{A. Notation}

In order to introduce the tetrad formulation for GR, we will use internal abstract indices as discussed, for instance, in [30-32]. For the convenience of the unfamiliar reader, we have included this short subsection with the basic ingredients. The more familiar reader can go directly to Eq. (4.2), where we introduce some new notation.

Let $\mathcal{V} \rightarrow M$ be a Minkowski vector bundle i.e., with typical fiber $V$, an $n$-dimensional vector space with internal indices $\{I, J, \ldots\}$, endowed with a Minkowskian metric $\eta$, which in abstract index notation reads $\eta_{I J}$. This metric allows us to define the $\eta$-metric volume form $\varepsilon$, the $\eta$-Hodge dual $\star_{\eta}$, and the $\eta$-trace $\operatorname{Tr}_{\eta}$ (see [33] for a recent review). The latter is given by

$$
\operatorname{Tr}_{\eta}(\alpha \wedge \beta)=\eta^{I_{1} J_{1}} \cdots \eta^{I_{1} J_{r}} \alpha_{I_{1} \cdots I_{r}} \wedge \beta_{J_{1} \cdots J_{r}} .
$$

Tetrads are bundle isomorphisms $T M \rightarrow \mathcal{V}$. In abstract index notation they look like $e_{\alpha}^{I}$, so it is clear that a tetrad, together with the nondegenerate metrics $g_{\alpha \beta}$ and $\eta_{I J}$, provides a way to swap space-time and internal indices. It is also clear from the index notation that tetrads can be identified with sections of the product bundle $\mathcal{V} \otimes T^{*} M$ i.e., elements of $\Gamma\left(\mathcal{V} \otimes T^{*} M\right)$, the set of $\mathcal{V}$-valued 1-forms over $M$, although in practice it would be more useful to identify them with $V$-valued 1-forms over $M$. This is the case, for instance, if $M$ is parallelizable (an example would be a noncompact manifold admitting a spinor structure [34]). In that case it is enough to work with coframes which, loosely speaking, are the local version of a tetrad. Indeed, a coframe $e_{\alpha}^{I}(p)$ at $p \in M$ is a linear ismorphism $T_{p} M \rightarrow V$ satisfying

$$
g_{\alpha \beta}(p)=\eta_{I J} e_{\alpha}^{I}(p) e_{\beta}^{J}(p) .
$$

Equivalently, a coframe can be thought of as an orthonormal basis in $T_{p}^{*} M$. When $M$ is parallelizable, coframes can be identified with global covector fields but, otherwise, they can only be considered as local covector fields [32].

It is also useful to introduce objects with more indices, the so-called generalized tensor fields $t \in \Gamma\left(\mathcal{V}^{\otimes p} \otimes \mathcal{V}^{* \otimes q} \otimes T M^{\otimes r} \otimes T^{*} M^{\otimes s}\right)$, where $\mathcal{V}^{*} \rightarrow M$ is the dual bundle of the Minkowski bundle. In index notation, generalized tensor fields look like $t_{\alpha_{1} \cdots \alpha_{s} I_{1} \cdots I_{q}}^{\beta_{1} \cdots \beta_{1} J_{1} \cdots J_{r}}$. We denote as $\Omega_{r}^{k}(M)$ the space of $k$-forms on $M$ with $r$ totally upper antisymmetric internal indices. Using Greek abstract indices $\{\alpha, \beta, \ldots\}$ for $M$ and capital Latin letters $\{I, J, \ldots\}$ for the internal indices, an element of $\Omega_{r}^{k}(M)$ will be written as

$$
\omega_{\alpha_{1} \cdots \alpha_{k}}^{I_{1} \cdots I_{r}}=\omega_{\left[\alpha_{1} \cdots \alpha_{k}\right]}^{\left[I_{1} \cdots I_{r}\right]}
$$

To ease the notation, space-time indices will often be omitted and we will simply write $\omega^{I_{1} \cdots I_{r}}$. Using the generalized Kronecker delta with internal indices, $\delta_{I_{1} \cdots I_{m}}^{J_{1} \cdots J_{m}}$, we define the graded wedge $\{\cdot \wedge \cdot\}: \Omega_{r}^{k}(M) \times \Omega_{s}^{m}(M) \rightarrow$ $\Omega_{r+s}^{k+m}(M)$

$$
\{A \wedge B\}_{I_{1} \cdots I_{r} J_{1} \cdots J_{s}}:=\frac{1}{r ! s !} \delta_{I_{1} \cdots I_{r} J_{1} \cdots J_{s}}^{K_{1} \cdots K_{r} L_{1} \cdots L_{s}} A_{K_{1} \cdots K_{r}} \wedge B_{L_{1} \cdots L_{S}}
$$

(this is analogous to the usual space-time wedge, although we have included curly brackets-not necessary, strictly speaking - as a reminder for those readers who may prefer explicit indices) and the graded bilinear product $[\cdot \AA \cdot]: \Omega_{r}^{k}(M) \times \Omega_{s}^{m}(M) \rightarrow \Omega_{r+s-2}^{k+m}(M)$

$$
\begin{aligned}
& {[A \wedge B]_{I_{1} \ldots I_{r-1} J_{1} \ldots J_{s-1}}} \\
& \quad:=\delta_{I_{1} \ldots I_{r-1} J_{1} \ldots J_{s-1}}^{K_{1} \ldots K_{r-1} A_{1} \ldots L_{r-1} M} A_{K_{1} \ldots K^{\prime}} \wedge B_{L_{1} \ldots L_{s-1}}^{M}
\end{aligned}
$$

where the symbol $\AA$ will be called the ledge (Lie wedge). The previous operation is performed by contracting the last index of $A$ with the first one of $B$ and antisymmetrizing the remaining ones. Although not necessary for our purposes, it is worth mentioning that $\Omega_{2}^{k}(M)$ with the ledge product

$$
[A \wedge B]_{I J}=A_{I K} \wedge B^{K}{ }_{J}-A_{J K} \wedge B^{K}{ }_{I}
$$

is a Lie algebra. Moreover, notice that $\{e \wedge e\}_{I J}=$ $2 e^{I} \wedge e^{J}$. The rest of the paper will use the following straightforward formula:

$$
\begin{gathered}
\operatorname{Tr}_{\eta}(\alpha \wedge\{\beta \wedge \gamma\})=-\operatorname{Tr}_{\eta}([\alpha \wedge \beta] \wedge \gamma), \\
\alpha \in \Omega_{2}^{*}(M), \quad \beta, \gamma \in \Omega_{1}^{*}(M) .
\end{gathered}
$$

\section{B. Constructing the geometric objects}

Consider $\left\{e_{\alpha}^{I}\right\}$ a coframe, such that $\varepsilon_{I_{1} \cdots I_{n}} e^{I_{1}} \wedge \cdots \wedge e^{I_{n}}$ defines a volume form on $M$. We define the Lorentzian $e$-metric

$$
g=\operatorname{Tr}_{\eta}(e \otimes e) \equiv g_{\alpha \beta}=\eta_{I J} e_{\alpha}^{I} e_{\beta}^{J}
$$

together with its $g$-Levi-Civita connection $\nabla$. We can now define the dual frame $\left\{E_{I}^{\alpha}\right\}$, given by the relations

$$
E_{I}^{\alpha} e_{\beta}^{I}=g_{\beta}^{\alpha}, \quad E_{I}^{\alpha} e_{\alpha}^{J}=\eta_{I}^{J}
$$

as well as the $e$-connection $\omega$ defined by

$$
\nabla_{X} E_{I}=\omega(X)^{K}{ }_{I} E_{K} \equiv \nabla_{\mu} E_{I}^{\alpha}=\omega_{\mu}{ }^{K}{ }_{I} E_{K}^{\alpha} \equiv \omega_{\mu}{ }^{K}{ }_{I}=e_{\alpha}^{K} \nabla_{\mu} E_{I}^{\alpha}
$$

which can be proven to uniquely determine $\omega$. Notice that the internal indices of $\omega$ are antisymmetric once the second 
one is raised $\omega_{\mu}^{I J}=-\omega_{\mu}^{J I}$. Notice also that we have the covariant derivative of forms over $\Omega_{s}^{r}(M)$ given by

$$
\mathcal{D} \alpha=\mathrm{d} \alpha+[\omega \wedge \alpha], \alpha \in \Omega_{s}^{r}(M)
$$

where here and in the following, we will sometimes omit the internal indices when not needed. Let us also denote the associated $e$ Christoffel as $\Gamma$, the $e$ torsion as $T$, the $e$ curvature as $R$, and the $\omega$ curvature as $F$, which are given by

$$
\begin{aligned}
\nabla_{E_{I}} E_{J} & =\Gamma^{K}{ }_{I J} E_{K} \equiv e^{I} \Gamma^{K}{ }_{I J}=\omega^{K}{ }_{J}, \\
\operatorname{Tor}\left(E_{I}, E_{J}\right) & =T^{L}{ }_{I J} E_{L} \equiv T^{L}{ }_{I J}=e^{L}\left(\operatorname{Tor}\left(E_{I}, E_{J}\right)\right), \\
\operatorname{Riem}\left(E_{I}, E_{J}\right) E_{K} & =R^{L}{ }_{K I J} E_{L} \equiv R^{L}{ }_{K I J} \\
& =e^{L}\left(\operatorname{Riem}\left(E_{I}, E_{J}\right) E_{K}\right), \\
F & =\mathrm{d} \omega+\frac{1}{2}[\omega \wedge \omega] \equiv F_{I J} \\
& =\mathrm{d} \omega_{I J}+\omega_{I K} \wedge \omega_{J}^{K},
\end{aligned}
$$

where Tor and Riem are the torsion and the Riemannian curvature of the $g$-Levi-Civita connection. In particular Tor $=0$. It is interesting to notice that, as mentioned in the introduction of this section, we are using $E_{I}^{\alpha}$ and $e_{\alpha}^{I}$ to

$$
\mathbb{S}_{\mathrm{GR}}^{(t)}(e)=\int_{M} L^{(t)}(e)-\int_{\partial_{L} M} \bar{\ell}^{(t)}(e),
$$

but suitably restricted to these spaces of tetrads. Notice that, essentially, we have only performed a "change of variables" from $g$ to $e$ through the (surjective but not injective) map

$$
\Phi(e)_{\alpha \beta}=\eta_{I J} e_{\alpha}^{I} e_{\beta}^{J} .
$$

Indeed, we have $\mathbb{S}^{(t)}=\Phi^{*} \mathbb{S}^{(m)}=\mathbb{S}^{(m)} \circ \Phi$. Since $\Phi$ is Lorentz invariant, the tetrad action is also Lorentz invariant i.e., if $\Psi \in S O(1,3)$, then $\mathbb{S}(\Psi . e)=\mathbb{S}(e)$. By using (4.4), it is possible to show that the Lagrangian admits the following explicit expression:

$$
\begin{aligned}
L^{(t)}(e) & =\frac{1}{2} \operatorname{Tr}_{\eta}\left(\left(F-\frac{\Lambda}{12}\{e \wedge e\}\right) \wedge \star_{\eta}\{e \wedge e\}\right) \\
& =\frac{1}{2} \varepsilon_{I J K L}\left(F^{I J}-\frac{\Lambda}{6} e^{I} \wedge e^{J}\right) \wedge e^{K} \wedge e^{L} .
\end{aligned}
$$

Finding a useful explicit expression for the boundary Lagrangian requires more work. Let us define the "internal" normal $N^{I}:=l_{\vec{\nu}} e^{I} \in \Omega_{1}^{0}(M)$, which satisfies $N_{I} \bar{e}_{\bar{\alpha}}^{I}=0$ and transform the space-time indices in Tor and Riem into internal indices in order to define $T$ and $R$. From the previous equations, the following relations are easy to derive:

$F_{J}^{I}=\frac{1}{2} R_{J K L}^{I} e^{K} \wedge e^{L}, \quad T_{I J}^{L}=\mathcal{D} e^{L}\left(E_{I}, E_{J}\right)$.

It is also straightforward to show that the following properties hold:

$$
\left(\mathcal{D}^{2} \alpha\right)_{I_{1} \cdots I_{r}}=[F \wedge \alpha]_{I_{1} \cdots I_{r}}, \quad \mathcal{D} F_{I J}=0, \quad \mathcal{D} e^{I}=0 .
$$

\section{Step 0: Action}

Let us consider the following spaces of nondegenerate tetrads on $M$ :

$$
\begin{aligned}
& \mathcal{F}_{N}^{(t)}=\left\{e \in \Omega_{1}^{1}(M) \mid e_{I} e^{I} \in \mathcal{F}_{N}^{(m)}\right\}, \\
& \mathcal{F}_{D}^{(t)}=\left\{e \in \Omega_{1}^{1}(M) \mid e_{I} e^{I} \in \mathcal{F}_{N}^{(m)} \text { and } \quad \bar{e}:=J^{*} e \text { fixed }\right\}
\end{aligned}
$$

that we will refer to as Neumann and Dirichlet tetrads, respectively. The actions $\mathbb{S}_{N}^{(t)}$ and $\mathbb{S}_{D}^{(t)}$ are both defined by the same expression

$$
\begin{aligned}
L^{(t)}(e) & :=L_{\mathrm{EH}}^{(m)}\left(\operatorname{Tr}_{\eta}(e \otimes e)\right), \\
\bar{\ell}^{(t)}(e) & :=\bar{\ell}_{\mathrm{GHY}}^{(m)}\left(\operatorname{Tr}_{\eta}(e \otimes e)\right)
\end{aligned}
$$

$N_{I} N^{I}=1$, and the "internal" projector $\gamma_{J}^{I}:=\eta_{J}^{I}-N^{I} N_{J}$. With these elements at hand, together with $\varepsilon_{I J K L}=$ $\operatorname{vol}_{\alpha \beta \gamma \delta} E_{I}^{\alpha} E_{J}^{\beta} E_{K}^{\gamma} E_{L}^{\delta}$ and (2.2), it is easy to rewrite $\bar{\ell}^{(t)}$ as

$$
\bar{\ell}^{(t)}(e)=-\varepsilon_{I J K L} N^{I} \overline{\mathcal{D}} N^{J} \wedge \bar{e}^{K} \wedge \bar{e}^{L}
$$

where

$$
\overline{\mathcal{D}} N^{J}=\mathrm{d} N^{J}+[\bar{\omega} \wedge N]^{J}=\mathrm{d} N^{J}+\bar{\omega}_{I}^{J} \wedge N^{I}
$$

is the induced covariant derivative over the boundary given by the pullback connection $\bar{\omega}=J^{*} \omega$. Recall also that $\bar{e}:=$ $J^{*} e$ which, in turn, allows us to define its dual $\bar{E}_{I}^{\bar{\alpha}}:=$ $\bar{g}^{\bar{\alpha}} \bar{\beta} \eta_{I J} \bar{e}_{\bar{\beta}}^{J}$ which satisfies

$$
\bar{E}_{I}^{\bar{\alpha}} \bar{e}_{\bar{\beta}}^{I}=\bar{g}_{\bar{\beta}}^{\bar{\alpha}}, \quad \bar{E}_{I}^{\bar{\alpha}} \bar{e}_{\bar{\alpha}}^{J}=\gamma_{I}^{K}
$$

With these ingredients, it is possible to obtain a better expression for $\bar{\ell}^{(t)}$ by considering 


$$
\begin{aligned}
& 2 \varepsilon_{I J K L}\left(\left(N^{I} \mathrm{~d} N^{J}-\frac{1}{2} \bar{\omega}^{I J}\right)-N^{I} \overline{\mathcal{D}} N^{J}\right) \wedge \bar{e}^{K} \wedge \bar{e}^{L} \\
& =-\varepsilon_{I J K L}\left(\bar{\omega}^{I J}+2 N^{I} \bar{\omega}^{J K} N_{K}\right) \wedge \bar{e}^{K} \wedge \bar{e}^{L}=-\varepsilon_{I J K L} \bar{\omega}^{K J}\left(\eta_{K}^{I}-2 N^{I} N_{K}\right) \wedge \bar{e}^{K} \wedge \bar{e}^{L} \\
& =-\varepsilon_{I J K L} \bar{\omega}^{K A}\left(\gamma_{A}^{J}+N^{J} N_{A}\right)\left(\gamma_{K}^{I}-N^{I} N_{K}\right) \wedge \gamma_{B}^{K} \bar{e}^{B} \wedge \gamma_{C}^{L} \bar{e}^{C} \\
& =-\gamma_{B}^{K} \gamma_{C}^{L} \varepsilon_{I J K L} \bar{\omega}^{K A}\left(\gamma_{A}^{J} \gamma_{K}^{I}-\left(\gamma_{A}^{J} N^{I} N_{K}-N^{J} N_{A} \gamma_{K}^{I}\right)-N^{J} N_{A} N^{I} N_{K}\right) \wedge \bar{e}^{B} \wedge \bar{e}^{C} \\
& =-\gamma_{B}^{K} \gamma_{C}^{L} \gamma_{A}^{J} \gamma_{K}^{I} \varepsilon_{I J K L} \bar{\omega}^{K A} \wedge \bar{e}^{B} \wedge \bar{e}^{C}
\end{aligned}
$$

which is zero as all four internal indices of $\varepsilon$ are projected. This identity allows us to obtain, as already done for instance in $[35,36]$, the alternative expression

$$
\begin{aligned}
\bar{\ell}^{(t)}(e) & =-\frac{1}{2} \operatorname{Tr}_{\eta}\left((\{N \wedge \mathrm{d} N\}-\bar{\omega}) \wedge \star_{\eta}\{\bar{e} \wedge \bar{e}\}\right) \\
& =-\frac{1}{2} \varepsilon_{I J K L}\left(2 N^{I} \mathrm{~d} N^{J}-\bar{\omega}^{I J}\right) \wedge \bar{e}^{K} \wedge \bar{e}^{L}
\end{aligned}
$$

\section{Step 1 and 2: Variations}

As before, we rely on the results and computations of the Appendix to obtain

$\mathbb{d} L^{(t)}=E_{I}^{(t)} \wedge \mathbb{d} e^{I}+\mathrm{d} \Theta^{(t)}, \mathbb{d} \bar{\ell}^{(t)}-\jmath^{*} \Theta^{(t)}=\bar{b}_{I}^{(t)} \wedge \mathbb{d} \bar{e}^{I}-\mathrm{d} \bar{\theta}^{(t)}$

where

$$
\begin{aligned}
E_{I}^{(t)}(e) & =-\left[\star_{\eta} F_{(\Lambda)} \wedge e\right]_{I}=\frac{1}{2} \varepsilon_{I J K L} F_{(\Lambda)}^{K L} \wedge e^{J}, \\
\bar{b}_{I}^{(t)}(e) & =\left[\star_{\eta}(\{N \wedge \mathrm{d} N\}-\bar{\omega}) \wedge \bar{e}\right]_{I}-2\left(\iota_{\bar{E}^{J}} \mathrm{~d} \bar{e}^{K}\right) \star_{\eta}\{N \wedge \bar{e}\}_{J K} N_{I} \\
& =\frac{1}{2} \varepsilon_{I J K L}\left(2 N^{K} \wedge \mathrm{d} N^{L}-\bar{\omega}^{K L}\right) \wedge \bar{e}^{J}-2\left(l_{\bar{E}^{J}} \mathrm{~d} \bar{e}^{K}\right) \varepsilon_{J K R S} N^{R} \wedge \bar{e}^{S} N_{I}, \\
\Theta^{(t)} & =\frac{1}{2} \operatorname{Tr}_{\eta}\left(\star_{\eta}\{e \wedge e\} \wedge \mathbb{d} \omega\right)=\frac{1}{2} \epsilon_{I J K L} e^{K} \wedge e^{L} \wedge \mathbb{d} \omega^{I J}, \\
\bar{\theta}^{(t)} & =\frac{1}{2} \operatorname{Tr}_{\eta}\left(\star_{\eta}\{\bar{e} \wedge \bar{e}\} \wedge\{N \wedge \mathbb{d} N\}\right)=\epsilon_{I J K L} \bar{e}^{K} \wedge \bar{e}^{L} \wedge N^{I} \wedge \mathbb{d} N^{J}, \\
F_{(\Lambda)}^{I J} & :=F^{I J}-\frac{\Lambda}{3} e^{I} \wedge e^{J}=\left(F-\frac{\Lambda}{6}\{e \wedge e\}\right)^{I J} .
\end{aligned}
$$

\section{E. Step 3: Space of solutions}

The solutions derived from $\mathbb{S}_{N}^{(t)}$ are nondegenerate tetrads $e \in \mathcal{F}_{N}^{(t)} \quad$ satisfying $E_{I}^{(t)}(e)=0$ and the "Neumann" boundary condition $\bar{b}_{I}^{(t)}(e)=0$. The solutions obtained from $\mathbb{S}_{D}^{(t)}$ are tetrads $e \in \mathcal{F}_{D}^{(t)}$ satisfying $E_{I}^{(t)}(e)=0$ and no additional condition at the boundary (the Dirichlet $\mathrm{BC}$ are part of the definition of $\mathcal{F}_{D}^{(t)}$ ).

\section{F. Step 4: Symplectic form}

Given a Cauchy embedding $\imath: \Sigma \hookrightarrow M$, we have

$$
\mathbb{\Omega}_{(t)}^{l}=\mathbb{d}\left(\int_{\Sigma} \iota^{*} \Theta^{(t)}-\int_{\partial \Sigma} \bar{\imath}^{*} \bar{\theta}^{(t)}\right) .
$$

Using the results of the previous section, we immediately obtain

$$
\begin{aligned}
\mathbb{\Omega}_{(t)}^{l}= & \frac{1}{2} \int_{\Sigma} \operatorname{Tr}_{\eta}\left(\star_{\eta} \mathbb{d}\{e \wedge e\} \mathbb{A} \mathbb{d} \omega\right) \\
& -\frac{1}{2} \int_{\partial \Sigma} \operatorname{Tr}_{\eta}\left(\star_{\eta} \mathbb{d}\{\bar{e} \wedge \bar{e}\} \mathbb{A}\{N \wedge \mathbb{d} N\}\right. \\
& \left.+\star_{\eta}\{\bar{e} \wedge \bar{e}\} \wedge\{\mathbb{d} N \mathbb{\wedge} \mathbb{d} N\}\right) .
\end{aligned}
$$

\section{G. Step 5: Charges}

A direct computation using the definition of the $\xi$-current $J_{\xi}^{(t)}$ leads to

$$
\begin{aligned}
J_{\xi}^{(t)}= & \frac{1}{2} \operatorname{Tr}_{\eta}\left(2\left[\star_{\eta} F_{(\Lambda)} \wedge e\right] \wedge l_{\xi} e-\star_{\eta} \mathcal{D}\{e \wedge e\}\right. \\
& \left.\wedge l_{\xi} \omega+\star_{\eta}\{e \wedge e\} \wedge\left(\mathcal{L}_{\xi}-\mathcal{L}_{\mathbb{X}_{\xi}}\right) \omega\right) \\
& -\operatorname{dTr}_{\eta}\left(\frac{1}{2} \star_{\eta}\{e \wedge e\} \wedge l_{\xi} \omega\right)
\end{aligned}
$$


where we have used $\iota_{\xi} F=\left(\mathcal{L}_{\xi}-\mathcal{D} l_{\xi}\right) \omega$, which follows from Cartan's magic formula. The second term of $J_{\xi}^{(t)}$ vanishes as the torsion $\mathcal{D} e$ is zero, while the third one is also zero as a consequence of (2.4) and the fact that the only background object is $\eta$, which is "constant." Thus we obtain

$$
\begin{aligned}
J_{\xi}^{(t)} & =\operatorname{Tr}_{\eta}\left(l_{\xi} e \wedge E^{(t)}\right)-\operatorname{dTr}_{\eta}\left(\frac{1}{2} \star_{\eta}\{e \wedge e\} \wedge l_{\xi} \omega\right) \\
\rightarrow Q_{\xi}^{(t)} & :=-\frac{1}{2} \operatorname{Tr}_{\eta}\left(\star_{\eta}\{e \wedge e\} \wedge l_{\xi} \omega\right) .
\end{aligned}
$$

$J_{\xi}^{(t)}$ is then exact over the space of solutions with $\xi$ potential $Q_{\xi}^{(t)}$. Similarly, we have for the boundary

$$
\begin{aligned}
\bar{J}_{\xi}^{(t)}-J^{*} Q_{\xi}^{(t)}= & -\frac{1}{2} \operatorname{Tr}\left(\left\{N \wedge\left(\mathcal{L}_{\xi}-\mathcal{L}_{{\aleph_{\xi}}_{\xi}}\right\} N\right) \wedge \star_{\eta}\{\bar{e} \wedge \bar{e}\}\right. \\
& \left.-2 \star_{\eta}(\{N \wedge \mathrm{d} N\}-\bar{\omega}) \wedge\left\{l_{\bar{\xi}} \bar{e} \wedge \bar{e}\right\}\right) \\
= & \operatorname{Tr}\left(0+\left[\star_{\eta}(\{N \wedge \mathrm{d} N\}-\bar{\omega}) \wedge \bar{e}\right] \wedge l_{\bar{\xi}} \bar{e}\right) \\
= & \operatorname{Tr}_{\eta}\left(l_{\bar{\xi}} \bar{e} \wedge \bar{b}^{(t)}\right) .
\end{aligned}
$$

In the last equality, a term may seem to be missing according to the definition of $\bar{b}^{(t)}$ but, in fact, such term vanishes as a consequence of $\bar{e}^{I} N_{I}=0$. We then conclude that the $\xi$ charges are given by

$$
\mathbb{Q}_{\xi,(t)}^{l}=\int_{\Sigma} \imath^{*} \operatorname{Tr}_{\eta}\left(l_{\xi} e \wedge E^{(t)}\right)-\int_{\partial \Sigma} \bar{l}^{*} \operatorname{Tr}_{\eta}\left(l_{\bar{\xi}} \bar{e} \wedge \bar{b}^{(t)}\right) .
$$

This expression, when pulled back to $\operatorname{Sol}\left(\mathbb{S}_{N}^{(t)}\right) \subset \mathcal{F}_{N}^{(t)}$, vanishes as expected because the theory is diffeomorphism invariant [15]. However, over $\operatorname{Sol}\left(\mathbb{S}_{D}^{(t)}\right) \subset \mathcal{F}_{D}^{(t)}$ the boundary integral will not be zero in general, as $\bar{b}^{(t)}$ does not necessarily vanish (fixing the tetrad on the boundary breaks the invariance under diffeomorphisms).

\section{H. Step 6: Comparing with the Hamiltonian formulation}

From step 6 of the CPS algorithm in the metric formalism and the following section, the equivalence is assured when no boundaries are present. The case with boundaries is again not as straightforward and it will be studied elsewhere.

\section{METRIC VERSUS TETRAD FORMULATION}

We have obtained in the previous sections the presymplectic structure over the space of solutions for both the metric and tetrad formulations together with their $\xi$ charges. In this section, we prove that the spaces can be naturally mapped, that their symplectic structures are equivalent (without considering the internal gauge freedom), and that the $\xi$ charges are equal.

\section{A. Space of solutions}

Let us first show the correspondence between the solution spaces of metric and tetrad gravity. To this end, we define the maps

$$
\Phi_{N}: \mathcal{F}_{N}^{(t)} \rightarrow \mathcal{F}_{N}^{(m)}, \quad \Phi_{D}: \mathcal{F}_{D}^{(t)} \rightarrow \mathcal{F}_{D}^{(m)}
$$

both obtained by assigning the following metric to a given tetrad

$$
\Phi(e)=\operatorname{Tr}_{\eta}(e \otimes e) \equiv \Phi(e)_{\alpha \beta}=\eta_{I J} e_{\alpha}^{I} e_{\beta}^{J} .
$$

Of course, in the Dirichlet case the compatibility condition $\bar{g}_{\bar{\alpha} \bar{\beta}}=\eta_{I J} \bar{e}_{\bar{\alpha}}^{I} \bar{e}_{\bar{\beta}}^{J}$ must hold for $\Phi_{D}$ to be well defined. On one hand, it is well known that $\Phi$ is surjective but not injective. For instance, $\Phi(-e)=\Phi(e)$. In fact, it can be proved that $\Phi(e)=\Phi\left(e^{\prime}\right)$ if and only if $e_{I}^{\prime}=\Psi_{I}^{J} e_{J}$ for some $\Psi \in S O(1,3)$.

On the other hand, we have that $\mathbb{S}^{(t)}=\mathbb{S}^{(m)} \circ \Phi$ so

$$
\mathbb{d}_{e} \mathbb{S}_{\mathrm{GR}}^{(t)}=\mathbb{d}_{e}\left(\mathbb{S}_{\mathrm{GR}}^{(m)} \circ \Phi\right)=\mathbb{d}_{\Phi(e)} \mathbb{S}_{\mathrm{GR}}^{(m)} \circ \mathbb{d}_{e} \Phi .
$$

It is easy to check that $\mathbb{d}_{e} \Phi$ is surjective. Hence, the relation between the spaces of solutions is clear: if $e \in \operatorname{Sol}\left(\mathbb{S}_{\mathrm{GR}}^{(t)}\right)$, then $\Phi(e) \in \operatorname{Sol}\left(\mathbb{S}_{\mathrm{GR}}^{(m)}\right)$ and if $g \in \operatorname{Sol}\left(\mathbb{S}_{\mathrm{GR}}^{(t)}\right)$, then every $e \in$ $\Phi^{-1}(\{g\})$ belongs to $\operatorname{Sol}\left(\mathbb{S}_{\mathrm{GR}}^{(t)}\right)$. This is equivalent to

$$
\operatorname{Sol}\left(\mathbb{S}_{\mathrm{GR}}^{(t)}\right)=\Phi^{-1} \operatorname{Sol}\left(\mathbb{S}_{\mathrm{GR}}^{(m)}\right) .
$$

\section{B. Presymplectic structures}

Let us now compare $\mathbb{\Omega}_{(m)}^{l}$ and $\mathbb{\Omega}_{(t)}^{l}$ by looking at their symplectic potentials. For that purpose, we perform the "change of variable" $g=\Phi(e):=e^{I} e_{I}$ in $\left(\Theta_{(m)}, \bar{\theta}_{(m)}\right)$. First, we notice that instead of working with $\Theta_{(m)}$, it is more convenient to work with its $g$ dual

$$
\left(\star_{g} \Theta_{(m)}\right)^{\mu}=\left(\star_{g} l_{\vec{W}} \operatorname{Vol}_{g}\right)^{\mu}=W^{\mu}
$$

where $W_{\mu}:=\nabla^{\beta} \mathbb{d} g_{\mu \beta}-g^{\alpha \beta} \nabla_{\mu} \mathbb{d} g_{\alpha \beta}$ was obtained in step 1 of Sec. III. Using the variation

$$
\begin{aligned}
& \mathbb{d} \omega_{\mu}^{K L}=\left(\mathbb{d} e_{\alpha}^{K}\right) \nabla_{\mu} E^{\alpha L}+E^{\alpha K} \nabla_{\mu} \mathbb{d} e_{\alpha}^{L} \\
& -\left(e_{\alpha I} \rrbracket e_{\beta}^{I}+e_{\beta I} \rrbracket e_{\alpha}^{I}\right) E^{\alpha K} \nabla_{\mu} E^{\beta L}-E^{\beta K} e_{\gamma}^{L}(\mathbb{} \rrbracket)_{\mu \beta}^{\gamma}
\end{aligned}
$$

which follows from the definition of $\omega_{\mu}^{I J}$, and the definition of $W^{\mu}$, we obtain on one hand

$$
W^{\mu}=2 E_{I}^{\mu} l_{E_{J}} \mathbb{d} \omega^{I J}+(\delta \mathcal{U})^{\mu}, \quad \mathcal{U}^{\alpha \mu}:=\left(g^{\beta \mu} E_{L}^{\alpha}-g^{\beta \alpha} E_{L}^{\mu}\right) \mathbb{d} e_{\beta}^{L}
$$


where $(\delta \mathcal{U})^{\mu}:=-\nabla_{\alpha} \mathcal{U}^{\alpha \mu}$ is the codifferential. On the other hand, a standard computation using the definition of the $g$-Hodge star operator leads to

$$
\left(\star_{g} \Theta^{(t)}\right)^{\mu}=2 E_{I}^{\mu} l_{E_{J}} \mathbb{\|} \omega^{I J} .
$$

Taking the Hodge dual of (5.3), using (5.2) and (5.4), and the fact that for $k$-forms we have $\star_{g^{\star}} g=(-1)^{k(n-k)+1} \mathrm{Id}$ and $\star_{g} \delta=(-1)^{k} \mathrm{~d} \star_{g}$, we finally obtain

$$
\Theta_{(m)}=\star_{g} W=\star_{g} \star_{g} \Theta^{(t)}+\star_{g} \delta \mathcal{U}=\Theta^{(t)}+\mathrm{d} \star_{g} \mathcal{U} .
$$

Once we have taken care of the bulk terms, we focus on the boundary ones. If we use $\varepsilon_{I J K L}=\operatorname{vol}_{\alpha \beta \gamma \delta} E_{I}^{\alpha} E_{J}^{\beta} E_{K}^{\gamma} E_{L}^{\delta}$, $N_{I}=\nu_{\alpha} E_{I}^{\alpha}$, and $\mathbb{} N^{I}=-N_{J} l_{E^{I}} \mathbb{} \rrbracket \bar{e}^{J}$, we can obtain on one hand

$$
\begin{aligned}
& \left(\bar{\theta}^{(t)}\right)_{\bar{\alpha} \bar{\beta}}=\varepsilon^{I J}{ }_{K L}\left(\bar{e}^{K} \wedge \bar{e}^{L}\right)_{\bar{\alpha} \bar{\beta}} N_{I} \llbracket N_{J} \\
& =-\left(\operatorname{vol}_{g}\right)^{\alpha \beta}{ }_{\gamma \delta} e_{\alpha}^{I} e_{\beta}^{J} E_{K}^{\gamma} E_{L}^{\delta}\left(\bar{e}_{\bar{\alpha}}^{K} \bar{e}_{\bar{\beta}}^{L}\right. \\
& \left.-\bar{e}_{\bar{\beta}}^{K} \bar{e}_{\bar{\alpha}}^{L}\right) \nu_{\sigma} E_{I}^{\sigma} N_{R} l_{E_{J}} \mathbb{d} \bar{e}^{R} \\
& =-2 N_{R}\left(J^{*} l_{\vec{\nu}} \operatorname{Vol}_{g}\right)_{\bar{\sigma} \bar{\alpha} \bar{\beta}} \bar{g}^{\bar{\sigma}} \bar{\mu}_{\mathbb{}} \bar{e}_{\bar{\mu}}^{R}=\left(l_{\vec{U}} \operatorname{Vol}_{\bar{g}}\right)_{\bar{\alpha} \bar{\beta}}
\end{aligned}
$$

where $\bar{U}^{\bar{\mu}}:=-2 N_{R} \bar{g}^{\bar{\mu}} \bar{\alpha}_{\mathbb{Q}} \bar{e}_{\bar{\alpha}}^{R}$. On the other hand, using (A8), we compute the following expression:

$$
\begin{aligned}
\bar{\theta}_{(m)}-\bar{\theta}^{(t)}-J^{*}\left(\star_{g} \mathcal{U}\right) & =l_{\vec{V}} \operatorname{vol}_{\bar{g}}-l_{\vec{U}} \operatorname{vol}_{\bar{g}}-\star_{\bar{g}} J^{*} l_{\vec{\nu}} \mathcal{U} \\
& =\imath\left(\vec{V}-\vec{U}-J^{*} l_{\vec{\nu}} \mathcal{U}\right) \operatorname{vol}_{g}
\end{aligned}
$$

where $\bar{V}_{\bar{\alpha}}:=-J_{\bar{\alpha}}^{\alpha}(l \vec{\nu} \llbracket g)_{\alpha}$ was obtained in the step 2 of Sec. III. Notice that we have made a small abuse of notation because $J^{*} l_{\nu} \mathcal{U}$ is a 1 -form so an index must be raised with the help of $\bar{g}$. Finally, we show that this last expression is in fact zero

$$
\begin{aligned}
& \bar{V}^{\bar{\alpha}}-\bar{U}^{\bar{\alpha}}-\bar{g}^{\bar{\alpha} \bar{\beta}} J_{\bar{\beta}}^{\beta} g_{\mu \beta} \nu_{\alpha} \mathcal{U}^{\alpha \mu} \\
& =-\bar{g}^{\bar{\alpha} \bar{\beta}} J_{\bar{\beta}}^{\beta} \nu^{\alpha} \eta_{I J}\left(e_{\alpha}^{I} \rrbracket e_{\beta}^{J}+e_{\beta}^{I} \rrbracket e_{\alpha}^{J}\right)+2 N_{R} \bar{g}^{\bar{\alpha} \bar{\beta}} \mathbb{\rrbracket} \bar{e}_{\bar{\beta}}^{R} \\
& -\bar{g}^{\bar{\alpha} \bar{\beta}} J_{\bar{\beta}}^{\beta} g_{\mu \beta} \nu_{\alpha}\left(g^{\sigma \mu} E_{L}^{\alpha}-g^{\sigma \alpha} E_{L}^{\mu}\right) \rrbracket e_{\sigma}^{L} \\
& =-N_{J} \bar{g}^{\bar{\alpha} \bar{\beta}} \mathbb{\rrbracket} \bar{e}_{\bar{\beta}}^{J}-\bar{g}^{\bar{\alpha} \bar{\beta}} \eta_{I J} \bar{e}_{\bar{\beta}}^{I} \nu^{\alpha} \mathbb{d} e_{\alpha}^{J}+2 N_{R} \bar{g}^{\bar{\alpha} \bar{\beta}} \mathbb{\rrbracket} \bar{e}_{\bar{\beta}}^{R} \\
& -\bar{g}^{\bar{\alpha} \bar{\beta}} N_{L} \rrbracket \bar{e}_{\bar{\beta}}^{L}+\bar{g}^{\bar{\alpha} \bar{\beta}} \nu^{\sigma} \eta_{L K} \bar{e}_{\bar{\beta}}^{K} \rrbracket e_{\sigma}^{L}=0
\end{aligned}
$$

which proves that

$$
\bar{\theta}_{(m)}=\bar{\theta}^{(t)}+J^{*}\left(\star_{g} \mathcal{U}\right) .
$$

Putting everything together, we finally obtain the main result of the paper

$$
\left(\Theta_{(m)}, \bar{\theta}_{(m)}\right)=\left(\Theta^{(t)}, \bar{\theta}^{(t)}\right)+\underline{\mathrm{d}}\left(\star_{g} \mathcal{U}, 0\right) .
$$

Hence, the symplectic potentials are equal up to a relative exact form. Notice that the fact that the left-hand side comes from the metric formalism while the right-hand side comes from the tetrad formalism is not a problem because the left-hand side is implicitly evaluated at the $e$-dependent metric $\Phi(e):=\eta_{I J} e^{I} e^{J}$.

Taking now the $\mathbb{d}$ exterior derivative of (5.6), integrating over a Cauchy slice $(\Sigma, \partial \Sigma)$, and using the relative Stokes's theorem (A2), we obtain the desired equality of the two presymplectic forms $\mathbb{\Omega}_{(t)}$ and $\mathbb{\Omega}_{(m)}$ (see Appendix A 1 for a brief account of the relative framework). More specifically, we have

$$
\mathbb{\Omega}_{(t)}=\Phi^{*} \Omega_{(m)} .
$$

A final comment is in order now: the previous formula says that both presymplectic structures are equivalent modulo the gauge freedom given by the kernel of $\Phi_{*}$. This gauge freedom is present in the tetrad formalism and originates in the $S O(1,3)$ invariance of $\Phi$ but it has no metric counterpart. In particular, this means that $\Omega_{(t)}$ has more degenerate directions than $\mathbb{\Omega}_{(m)}$. This can be neatly understood by noticing that if we consider a curve $\Psi_{\tau} \in S O(1,3)$ and its associated vector $\mathbb{V}_{(t)}:=\left.\frac{\mathrm{d}}{\mathrm{d} \tau}\right|_{0} \Psi_{\tau} \cdot e \in T_{e} \mathcal{F}^{(t)}$, we have

$$
\begin{aligned}
\Psi_{*} \mathbb{V}_{(t)} & =\left.\frac{\mathrm{d}}{\mathrm{d} \tau}\right|_{0} \Phi\left(\Psi_{\tau} \cdot e\right)=\left.\frac{\mathrm{d}}{\mathrm{d} \tau}\right|_{0} \Phi(e)=0 \rightarrow l_{\mathbb{V}_{(t)}} \mathbb{\Omega}_{(t)} \\
& =l_{\mathbb{V}_{(t)}} \Phi^{*} \Omega_{(m)}=\Phi^{*}\left(l_{\Phi_{*} \mathbb{V}_{(t)}} \mathbb{\Omega}_{(m)}\right)=0 .
\end{aligned}
$$

$\mathbb{V}_{(t)}$ is a nonzero vector which belongs to the kernel of $\Omega_{(t)}$ (as a consequence of being in the kernel of $\Psi_{*}$ ) so it is a gauge vector field. However, the metric counterpart $\mathbb{V}_{(m)}:=$ $\Psi_{*} \mathbb{V}=0$ is not gauge because by definition the zero vector is not gauge. Finally, the equivalence of the charges (3.4) and (4.8) is obvious as both theories are equivalent, implying that $\bar{b}_{(m)}$ and $\bar{b}^{(t)}$ are equivalent as well.

\section{CONCLUSIONS}

In this paper, we have studied the metric and tetrad formulations for general relativity on a manifold with boundary. By considering the appropriate bulk and boundary Lagrangians, we have shown that both theories are equivalent and hence, as one would expect, they are symplectically equivalent in the covariant phase space. Here we have focused on Dirichlet and Neumann BC, but any other $\mathrm{BC}$ will give the same results as long as the metric and tetrad actions are in a suitable correspondence.

It has been known for some time that, in the absence of boundaries, the metric symplectic current $\Omega_{(m)}:=\mathbb{d} \Theta_{(m)}$ is equal to the tetrad symplectic current $\Omega^{(t)}:=\mathbb{d} \Theta^{(t)}$ up to an exact form $\mathrm{d} A$ (and thus cohomologically equal). Therefore, their presymplectic forms over the space of solutions are equivalent since the integral of $\mathrm{d} A$ over a 
Cauchy slice $\Sigma$ is zero according to Stokes's theorem. On the other hand, if one considers a space-time with boundaries, the boundary-free covariant phase space procedure fails. This is due to some ambiguities that arise in the construction of the presymplectic form that hinder the direct comparison between metric and tetrad formulations. This has caused some discrepancies in previous works $[20,22,23]$, but as we have shown, these difficulties arise because the traditional covariant phase space methods are only suited for the boundary-free case.

When boundaries are present, it is necessary to use more sophisticated techniques, like the relative bicomplex framework [15]. Following the ideas of that formalism, we obtain the main result of the paper: the metric symplectic currents $\left(\Omega_{(m)}, \bar{\omega}_{(m)}\right):=\left(\mathbb{} \Theta_{(m)}, \mathbb{d} \bar{\theta}_{(m)}\right)$ and the tetrad symplectic currents $\left(\Omega^{(t)}, \bar{\omega}^{(t)}\right):=\left(\mathbb{\Phi} \Theta^{(t)}, \mathbb{\Phi} \bar{\theta}^{(t)}\right)$ are equal up to a relative exact form $\mathrm{d}(A, \bar{a})$. This implies, in particular, that they are equal in the relative cohomology (see Appendix A 1 for the relevant definitions) and that their presymplectic forms over the space of solutions are equivalent. Indeed, the relative Stokes's theorem tells us that the integral of $\underline{\mathrm{d}}(A, \bar{a})$ over a relative Cauchy slice $(\Sigma, \partial \Sigma)$ is zero. Furthermore, we have proved that the Noether charges are equivalent as expected. Finally, we have applied the covariant phase space methods to the asymptotically flat case to recover the well-known formula for the ADM energy.

\section{ACKNOWLEDGMENTS}

The authors wish to thank Abhay Ashtekar, Laurent Freidel, and Simone Speziale for correspondence that prompted us to clarify some of the points discussed in the paper. This work has been supported by the Spanish Ministerio de Ciencia Innovación y Universidades-Agencia Estatal de Investigación FIS2017-84440-C2-2-P grant. J. M.-B. is supported by the Eberly Research Funds of
Penn State, by the NSF Grant No. PHY-1806356 and by the Urania Stott fund of Pittsburgh foundation UN2017-92945. E. J. S. V. is supported by the Madrid Government (Comunidad de Madrid-Spain) under the Multiannual Agreement with $\mathrm{UC} 3 \mathrm{M}$ in the line of Excellence of University Professors (EPUC3M23), and in the context of the V PRICIT (Regional Programme of Research and Technological Innovation).

\section{APPENDIX: ANCILLARY MATERIAL}

\section{Mathematical background \\ a. Relative bicomplex framework}

In this section we include a summary of the definitions and results of [15]. Consider an $n$-dimensional manifold $M$ with boundary $\partial M$ (possibly empty) and a space of fields $\mathcal{F}$ defined on it (sections of a bundle $E \rightarrow M$ ). The geometric structure of $\mathcal{F}$ may be understood by studying the infinite jet space of $E$. However, it is also possible to deal with $\mathcal{F}$ as is customary in the physics literature: by considering it as an infinite dimensional manifold endowed with standard operations such as the exterior derivative $\mathbb{d}$, the interior product $l$, or the Lie derivative $\mathcal{L}$. Physical field theories are described in terms of locally constructed fields over the space $M \times \mathcal{F}$, a space consisting of points of $M$ and fields over $M$.

We define the relative pair $(M, N)$ with $N \subset M$ being a submanifold $N \stackrel{J}{\hookrightarrow} M$ of codimension 1 of $M$. In this paper, we will always assume $N \subset \partial M$. Then, we have that the relative boundary of the pair is defined as

$$
\underline{\partial}(M, N):=(\partial M \backslash N, \partial N)
$$

which satisfies $\underline{\partial}^{2}=0$ and $\underline{\partial}(M, \partial M)=\varnothing$. The space of relative forms and the generalizations of some familiar operators to the present case are defined as

$$
\begin{aligned}
\Omega^{k}(M, N) & :=\Omega^{k}(M) \oplus \Omega^{k-1}(N), \quad \underline{\mathrm{d}}(A, \bar{a}):=\left(\mathrm{d} A, J^{*} A-\mathrm{d} \bar{a}\right), \\
\underline{l}_{V}(A, \bar{a}) & :=\left(l_{V} A,-l_{\bar{V}} \bar{a}\right), \quad \underline{\star}_{g}(A, \bar{a}):=\left(\star_{g} A, \star_{\bar{g}} \bar{a}\right), \\
\underline{f}^{*}(A, \bar{a}) & =\left(f^{*} A,\left(\left.f\right|_{N}\right)^{*} \bar{a}\right), \quad \underline{\mathbb{Q}}(A, \bar{a})=(\mathbb{d} A, \mathbb{d} \bar{a}),
\end{aligned}
$$

where $\bar{V}:=\left.V\right|_{N}$ has to be tangent to $N$. Notice that $\underline{\mathrm{d}}^{2}=0$, hence, we can define the so called relative cohomology $H^{k}(M, N)$. Two classes $\left[\left(A_{1}, \bar{a}_{1}\right)\right],\left[\left(A_{2}, \bar{a}_{2}\right)\right] \in H^{k}(M, N)$ are equal if and only if there exists $(B, \bar{b}) \in \Omega^{k-1}(M, N)$ such that $\left(A_{1}, \bar{a}_{1}\right)=\left(A_{2}, \bar{a}_{2}\right)+\mathrm{d}(B, \bar{b})$. The integral of a relative top-form $(A, \bar{a}) \in \Omega^{n}(M, N)$ over the relative pair $(M, N)$ is defined as

$$
\int_{(M, N)}(A, \bar{a}):=\int_{M} A-\int_{N} \bar{a} .
$$

We have the relative Stokes's theorem given by

$$
\int_{(M, N)} \underline{\mathrm{d}}(B, \bar{b})=\int_{\underline{\partial}(M, N)} \underline{J}^{*}(B, \bar{b})
$$

which in turn implies that (A1) for $N=\partial M$ is well defined on relative cohomology because $\underline{\partial}(M, \partial M)=\varnothing$. We introduce now the space of forms $\mathbb{\Omega}^{(r, s)}(M \times \mathcal{F})$ of degree $r$ in $M$ (horizontal part) and $s$ in $\mathcal{F}$ (vertical part). Endowed with the wedge product $\mathbb{A}$, this space becomes a bigraded algebra with two exterior derivatives: the horizontal d, 
which increases $r$ in one unit, and the vertical $\mathbb{d}$, increasing $s$ in one unit. The wedge product $\mathbb{\wedge}$ restricted to $(k, 0)$ forms coincides with $\wedge$. We will often abuse notation and use the latter. If we replace $(M, \mathrm{~d})$ by the relative pair $((M, N), \underline{\mathrm{d}})$, we can define the relative bicomplex

$\mathbb{\Omega}_{\mathrm{loc}}^{(r, s)}((M, N) \times \mathcal{F})=\mathbb{\Omega}_{\mathrm{loc}}^{(r, s)}(M \times \mathcal{F}) \oplus \mathbb{\Omega}_{\mathrm{loc}}^{(r-1, s)}(N \times \mathcal{F})$

where the loc subscript indicates that we only consider $(r, s)$-forms which are locally constructed i.e., a form $\alpha$ evaluated at $p$ only depends on $p, \phi(p)$, and finitely many of the derivatives of $\phi$ at $p$.

\section{b. Lagrangians and actions}

Definition A.3 We define a Lagrangian pair as an element of

$$
\operatorname{Lag}(M):=\mathbb{\Omega}_{\mathrm{loc}}^{(n, 0)}((M, \partial M) \times \mathcal{F})
$$

Remember that

$$
\left[\left(L_{1}, \bar{\ell}_{1}\right)\right]=\left[\left(L_{2}, \bar{\ell}_{2}\right)\right] \equiv\left(L_{2}, \bar{\ell}_{2}\right)=\left(L_{1}, \bar{\ell}_{1}\right)+\underline{\mathrm{d}}(Y, \bar{y}) \equiv \begin{aligned}
& L_{2}=L_{1}+\mathrm{d} Y \\
& \bar{\ell}_{2}=\bar{\ell}_{1}+J^{*} Y-\mathrm{d} \bar{y}
\end{aligned} .
$$

Definition A.4 A local action is a map $\mathbb{S}: \mathcal{F} \rightarrow \mathbb{R}$ of the form

$$
\mathbb{S}(\phi)=\int_{(M, \partial M)}(L, \bar{\ell})(\phi)
$$

for some local Lagrangian pair $(L, \bar{\ell}) \in \operatorname{Lag}(M)$.

Definition A.6 $\left(L_{i}, \bar{\ell}_{i}\right) \in \operatorname{Lag}(M)$ are $\int$-equivalent, which we denote as $\left(L_{1}, \bar{\ell}_{1}\right) f\left(L_{2}, \bar{\ell}_{2}\right)$, if for every $\phi \in \mathcal{F}$, we have

$$
\int_{(M, \partial M)}\left(L_{1}, \bar{\ell}_{1}\right)(\phi)=\int_{(M, \partial M)}\left(L_{2}, \bar{\ell}_{2}\right)(\phi) .
$$

In this work, we have only considered contractible bundles, for which the $\int$ equivalence is the same as the cohomological equivalence (a proof was given in [15]). Nevertheless, if the bundles are not contractible, it is still possible to keep track of the ambiguities that arise from the fact that there exist nonzero Lagrangians $[(L, \bar{\ell})] \neq 0$ whose Euler-Lagrange equations and $\mathrm{BC}$ are zero.

\section{c. Variations}

We assume that the action is defined in such a way that it is possible to find Euler-Lagrange equations and boundary equations $(E, \bar{b})$, and symplectic potentials $(\Theta, \bar{\theta})$, such that

$$
\mathbb{d} L=E_{r} \wedge \mathbb{d} \phi^{r}+\mathrm{d} \Theta, \quad \mathbb{d} \bar{\ell}-J^{*} \Theta=\bar{b}_{r} \wedge \mathbb{d} \phi^{r}-\mathrm{d} \bar{\theta}
$$

where $r$ labels the fields of the theory $\phi=\left(\phi^{1}, \ldots, \phi^{R}\right) \in \mathcal{F}$. If this is not possible, the theory is ill posed and we have to change the space of fields $\mathcal{F}$ and/or the action $\mathbb{S}$. The symplectic potentials $(\Theta, \bar{\theta})$ are defined up to a relative exact form. The space of solutions is

$$
\operatorname{Sol}(\mathbb{S}):=\{\phi \in \mathcal{F} \mid(E, \bar{b})(\phi)=0\} .
$$

\section{d. Symplectic structure}

We define the symplectic currents as $(\Omega, \bar{\omega}):=\mathbb{d}(\Theta, \bar{\theta})$. The relevant object is the relative integral of the symplectic currents over a Cauchy embedding $\imath:(\Sigma, \partial \Sigma) \hookrightarrow$ $\left(M, \partial_{L} M\right)$

$$
\mathbb{\Omega}_{\mathbb{S}}^{l}:=\int_{(\Sigma, \partial \Sigma)} \underline{\mathbb{d}} \underline{\imath}^{*}(\Theta, \bar{\theta}) \in \mathbb{\Omega}^{2}(\mathcal{F}) .
$$

It can be proved that the pullback of $\Omega_{\mathbb{S}}^{l}$ to the space of solutions is independent of the Cauchy embedding, endowing $\operatorname{Sol}(\mathbb{S})$ with a presymplectic structure canonically associated with $\mathbb{S}$.

\section{e. Currents and charges}

Given some vector field $\xi^{\alpha}$ tangent to the lateral boundary, we define the $\xi$ currents and the $\xi$ charges as

$$
\begin{aligned}
\left(J_{\xi}, \bar{J}_{\xi}\right) & :=\underline{l}_{\xi}(L, \bar{\ell})-\underline{l}_{\mathbb{X}_{\xi}}(\Theta, \bar{\theta}), \\
\mathbb{Q}_{\xi}^{l} & :=\int_{(\Sigma, \partial \Sigma)} \underline{\underline{l}}^{*}\left(J_{\xi}, \bar{J}_{\xi}\right) \in l^{0}(\mathcal{F}) .
\end{aligned}
$$

The $\xi$ charges in general depend on the chosen Lagrangians and on the embedding. If we compare the $\xi$ charges associated with two embeddings we obtain the following flux law:

$$
\begin{aligned}
\mathbb{Q}_{\xi}^{l_{2}}-\mathbb{Q}_{\xi}^{l_{1}^{1}}= & \int_{\left(N, \partial_{L} N\right)}\left(E_{r}, \bar{b}_{r}\right)(\phi) \mathcal{L}_{\xi} \phi^{r} \\
& +\int_{\left(N, \partial_{L} N\right)}\left(\mathcal{L}_{\xi}-\underline{\mathcal{L}}_{\mathbb{X}_{\xi}}\right)(L, \bar{\ell})
\end{aligned}
$$

where $N$ is the manifold bounded by the Cauchy slices $l_{1}(\Sigma)$ and $\iota_{2}(\Sigma)$. In general, the charge $\mathbb{Q}_{\xi}^{l}$ is not the Hamiltonian of the vector field $\mathbb{X}_{\xi}$ because 


$$
\begin{aligned}
\mathbb{d} \mathbb{Q}_{\xi}^{l}= & \left.l_{\mathbb{X}_{\xi}} \mathbb{R}_{\mathbb{S}}^{l}+\int_{(\Sigma, \partial \Sigma)} \underline{\underline{l}^{*}(\underline{\underline{l} \xi}}\left(E_{r}, \bar{b}_{r}\right) \wedge \mathbb{d} \phi^{r}\right) \\
& +\int_{(\Sigma, \partial \Sigma)} \underline{\underline{*}}^{*}\left(\mathcal{L}_{\xi}-\mathcal{L}_{\mathbb{X}_{\xi}}\right)(\Theta, \bar{\theta})
\end{aligned}
$$

The $\xi$ charge is the Hamiltonian of $\mathbb{X}_{\xi}$ over $\left(\operatorname{Sol}(\mathbb{S}), \mathbb{\Omega}_{\mathbb{S}}\right)$ if and only if the last integral vanishes.

\section{Some computations in the metric case}

Let us start off with a list of some of the well-known variations of the relevant objects used in the metric formalism

$$
(\mathbb{d} \nabla)_{\beta \gamma}^{\alpha}=\frac{1}{2} g^{\alpha \mu}\left(\nabla_{\beta} \llbracket g_{\mu \gamma}+\nabla_{\gamma} \mathbb{d} g_{\beta \mu}-\nabla_{\mu} \mathbb{d} g_{\beta \gamma}\right),
$$

$$
\begin{aligned}
& \mathbb{d} \nu_{\alpha}=\frac{1}{2} \nu^{\beta} \nu^{\gamma} \mathbb{d} g_{\beta \gamma} \nu_{\alpha}, \\
& \mathbb{d} \operatorname{Ric}_{\beta \gamma}=\nabla_{\alpha}(\mathbb{d} \nabla)_{\beta \gamma}^{\alpha}-\nabla_{\beta}(\mathbb{d} \nabla)^{\alpha}{ }_{\alpha \gamma},
\end{aligned}
$$

$$
\begin{gathered}
\mathbb{d}=-\mathrm{Ric}^{\alpha \beta} \mathbb{d} g_{\alpha \beta}+\nabla^{\alpha} \nabla^{\beta} \mathbb{d} g_{\alpha \beta}-\nabla^{\alpha} \nabla_{\alpha} \llbracket g, \\
\mathbb{d} \operatorname{vol}_{g}=\frac{\mathbb{d} g}{2} \operatorname{vol}_{g},
\end{gathered}
$$

$$
\begin{aligned}
\mathbb{d} K_{\bar{\alpha} \bar{\beta}}= & \frac{1}{2}\left(K_{\bar{\alpha} \bar{\beta}} \nu^{\alpha} \nu^{\beta} \llbracket g_{\alpha \beta}+J_{\bar{\alpha}}^{\alpha} J_{\bar{\beta}}^{\beta}\left(\nabla_{\bar{\nu}} \rrbracket g_{\alpha \beta}\right.\right. \\
& \left.\left.-\nu^{\mu} \nabla_{\alpha} \rrbracket g_{\mu \beta}-\nu^{\mu} \nabla_{\beta} \llbracket g_{\mu \alpha}\right)\right),
\end{aligned}
$$

where $\mathbb{d} g:=g^{\alpha \beta} \mathbb{d} g_{\alpha \beta}$ stands for the $g$ trace of $\mathbb{d} g_{\alpha \beta}$ (not to be confused with the variation of the determinant of $g$, which we do not use in this paper). Besides, we use the notation $\left(\iota_{\vec{\nu}} \mathbb{d}\right)_{\beta}:=\nu^{\alpha} \mathbb{d} g_{\alpha \beta}$.

\section{a. Variations}

With those variations and the Lagrangians $\left(L_{\mathrm{EH}}^{(m)}, \bar{\ell}_{\mathrm{GHY}}^{(m)}\right)=\left((R-2 \Lambda) \operatorname{vol}_{g},-2 K \operatorname{vol}_{\bar{g}}\right)$, we have

$$
\mathbb{d}\left(L_{\mathrm{EH}}^{(m)}, \bar{\ell}_{\mathrm{GHY}}^{(m)}\right)=\left(E_{(m)}, \bar{b}_{(m)}\right) \wedge \mathbb{d} g+\underline{\mathrm{d}}\left(\Theta_{(m)}, \bar{\theta}_{(m)}\right)
$$

where

$$
\begin{aligned}
E_{(m)}^{\alpha \beta} & =\left(\left(\frac{R}{2}-\Lambda\right) g^{\alpha \beta}-\operatorname{Ric}^{\alpha \beta}\right) \operatorname{vol}_{g}, \quad \bar{b}_{(m)}^{\bar{\alpha} \bar{\beta}}=\left(K^{\bar{\alpha} \bar{\beta}}-K \bar{g}^{\bar{\alpha} \bar{\beta}}\right) \operatorname{vol}_{\bar{g}}, \Theta_{(m)}=l_{\vec{W}} \operatorname{Vol}_{g}, \quad \bar{\theta}_{(m)}=l_{\vec{V}} \operatorname{Vol}_{\bar{g}}, \\
W^{\alpha} & =\left(g^{\alpha \mu} g^{\beta \lambda}-g^{\alpha \lambda} g^{\beta \mu}\right) \nabla_{\lambda} \mathbb{d} g_{\beta \mu}, \quad \bar{V}^{\bar{\alpha}}=-\bar{g}^{\bar{\alpha} \bar{\beta}} g^{\alpha \lambda} J_{\bar{\beta}}^{\beta} \nu_{\lambda} \mathbb{d} g_{\alpha \beta} .
\end{aligned}
$$

\section{b. Symplectic form}

The symplectic currents are given by

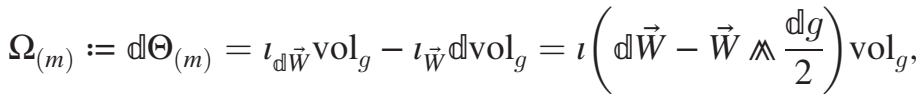

$$
\begin{aligned}
& \bar{\omega}_{(m)}:=\mathbb{d} \bar{\theta}_{(m)}=l_{\mathbb{d} \vec{V}} \operatorname{Vol}_{\bar{g}}-l_{\vec{V}} \mathbb{d} \operatorname{vol}_{\bar{g}}=\imath\left(\mathbb{d} \vec{V}-\vec{V} \Uparrow \frac{\mathbb{d} \bar{g}}{2}\right) \operatorname{vol}_{\bar{g}} .
\end{aligned}
$$

To ease the notation, here we are using the parenthesis for the interior product instead of a subscript. These terms can then be rewritten as follows: 


$$
\begin{aligned}
& g_{\alpha \beta}\left(\mathbb{d} W^{\beta}-W^{\beta} \mathbb{\mathfrak { A }} \frac{\mathbb{d} g}{2}\right)=\frac{1}{2} \mathbb{d} g_{\alpha \mu} \mathbb{\wedge} \nabla^{\mu} \mathbb{d} g+\frac{1}{2} g^{\sigma \beta} g^{\mu \lambda} \mathbb{d} g_{\sigma \mu} \mathbb{\wedge} \nabla_{\alpha} \mathbb{d} g_{\beta \lambda}+\frac{1}{2} \mathbb{d} g \mathbb{\Re} \nabla^{\beta} \mathbb{d} g_{\alpha \beta}-\frac{1}{2} \mathbb{d} g \mathbb{\Re} \nabla_{\alpha} \mathbb{d} g-g^{\mu \lambda} \mathbb{d} g_{\beta \mu} \mathbb{\wedge} \nabla^{\beta} \mathbb{d} g_{\alpha \lambda}
\end{aligned}
$$

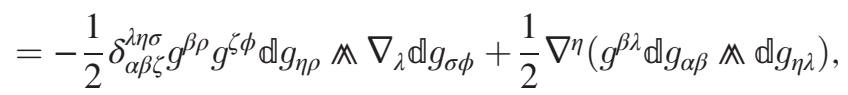

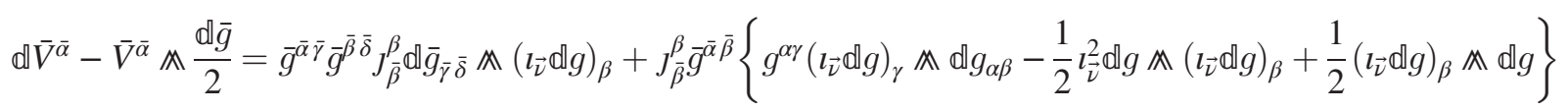

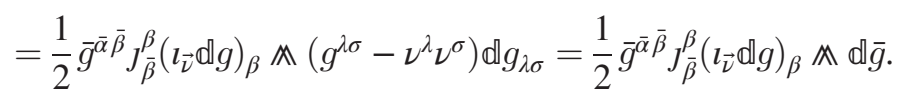

Consider a Cauchy embedding $l: \Sigma \hookrightarrow M$, where we have the $g$-normal $n^{\alpha}$ to $l(\Sigma) \subset M$, the $\bar{g}$-normal $\bar{m}^{\bar{\alpha}}$ to $\imath(\partial \Sigma) \subset \partial_{L} M$, the $\gamma$-normal $\mu^{b}$ to $\partial \Sigma \subset \Sigma$, and the induced metric $\gamma=\imath^{*} g$. We denote also $m^{\alpha}:=J_{\bar{\alpha}}^{\alpha} \bar{m}^{\bar{\alpha}}$ and $\mu_{b}^{\beta}:=\iota_{b}^{\beta} \mu^{b}$. Integrating the symplectic current over $(\Sigma, \partial \Sigma)$ we obtain the presymplectic form

$$
\begin{aligned}
& \mathbb{\Omega}_{(m)}^{l}=\int_{(\Sigma, \partial \Sigma)} \underline{\underline{l}}^{*}\left(\Omega_{(m)}, \bar{\omega}_{(m)}\right) \underset{(\mathrm{III} .3)}{\stackrel{(\mathrm{II} .2)}{=}} \\
& =-\int_{\Sigma} n_{\alpha}\left(\mathbb{d} W^{\alpha}-W^{\alpha} \mathbb{\Uparrow} \frac{\mathbb{d} g}{2}\right) \operatorname{vol}_{\gamma}-\int_{\partial \Sigma} \bar{m}_{\bar{\alpha}}\left(\mathbb{d} \bar{V}^{\bar{\alpha}}-\bar{V}^{\bar{\alpha}} \mathbb{A} \frac{\mathbb{d} \bar{g}}{2}\right) \operatorname{vol}_{\bar{\gamma}} \\
& =\frac{1}{2} \int_{\Sigma} n^{\alpha} \delta_{\alpha \beta \zeta}^{\lambda \eta \sigma} g^{\beta \rho} g^{\zeta \phi} \mathbb{d} g_{\eta \rho} \mathbb{\wedge} \nabla_{\lambda} \mathbb{d} g_{\sigma \phi} \operatorname{vol}_{\gamma}-\frac{1}{2} \int_{\partial \Sigma}\left(n^{\alpha} g^{\beta \eta} \mu^{\lambda}+m^{\alpha} \nu^{\beta} \bar{g}^{\bar{\eta}} \bar{\lambda} J_{\bar{\eta}}^{\eta} J_{\bar{\lambda}}^{\lambda}\right) \mathbb{d} g_{\alpha \beta} \mathbb{\wedge} \mathbb{d} g_{\eta \lambda} \operatorname{vol}_{\bar{\gamma}}
\end{aligned}
$$

Notice that we have used Stokes's theorem, Gauss's lemma (to write the covariant derivative $\nabla$ of $M$ in terms of the covariant derivative $D$ of $\Sigma$ and its extrinsic curvature) together with the fact that $T_{\alpha \eta}:=g^{\beta \lambda} \mathbb{d} g_{\alpha \beta} \mathbb{A} \mathbb{d} g_{\eta \lambda}$ is antisymmetric (which kills the extrinsic curvature terms) to take $n_{\alpha} \nabla_{\eta} T^{\alpha \eta}$ to the boundary.

\section{c. Charges}

The $\xi$ currents are given by

$$
\begin{aligned}
& \left(J_{\xi}^{(m)}, \bar{J}_{\xi}^{(m)}\right)=\underline{l}_{\xi}\left(L_{\mathrm{EH}}^{(m)}, \bar{\ell}_{\mathrm{GHY}}^{(m)}\right)-\underline{l}_{\mathbb{\zeta}_{\xi}}\left(\Theta_{(m)}, \bar{\theta}_{(m)}\right)=\left(l_{\vec{\xi}} L_{\mathrm{EH}}^{(m)}, l_{\vec{\xi}} \bar{\epsilon}_{\mathrm{GHY}}^{(m)}\right)-\left(l_{\vec{W}} \operatorname{Vol}_{g}, l_{\vec{V}} \operatorname{Vol}_{\bar{g}}\right) \\
& =\left((R-2 \Lambda) l_{\vec{\xi}} \operatorname{Vol}_{g}, 2 K l_{\vec{\xi}} \operatorname{Vol}_{\bar{g}}\right)-\left(l_{\underline{l}_{X_{\bar{\xi}}} \vec{W}} \operatorname{Vol}_{g}, l_{\underline{l}_{X_{\bar{\xi}}} \vec{V}} \operatorname{Vol}_{\bar{g}}\right)
\end{aligned}
$$

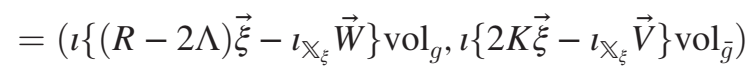

$$
\begin{aligned}
& =\left(\star_{g}\left\{(R-2 \Lambda) \xi-l_{\mathbb{X}_{\bar{\xi}}} W\right\}, \star_{\bar{g}}\left\{2 K \bar{\xi}-l_{\mathbb{X}_{\bar{\xi}}} \bar{V}\right\}\right) .
\end{aligned}
$$

Using the definition of the Ricci and Riemann tensor together with (2.4), we obtain

$$
(R-2 \Lambda) \xi_{\alpha}-l_{\mathbb{X}_{\xi}} W_{\alpha}=(\delta \mathrm{d} \xi)_{\alpha}+2\left(l_{\tilde{\xi}} \tilde{E}\right)_{\alpha}
$$

where $\delta$ is the codifferential (which is equal to minus the divergence) and $\tilde{E}_{(m)}^{\alpha \beta}:=E_{(m)}^{\alpha \beta} / \operatorname{vol}_{g}$ (the prefactor multiplying the volume form in $\left.E_{(m)}\right)$. Meanwhile, at the boundary

$$
\begin{aligned}
2 K \bar{\xi}^{\bar{\alpha}}-l_{\mathbb{X}_{\xi}} \bar{V}^{\bar{\alpha}} & =2 K \bar{\xi}^{\bar{\alpha}}+\bar{g}^{\bar{\alpha} \bar{\beta}} J_{\bar{\beta}}^{\beta} \nu^{\alpha}\left(\nabla_{\alpha} \xi_{\beta}+\nabla_{\beta} \xi_{\alpha}\right) \stackrel{\vec{\xi}\lrcorner \vec{\nu}}{=} 2 K \bar{\xi}^{\bar{\alpha}}+\bar{g}^{\bar{\alpha} \bar{\beta}} J_{\bar{\beta}}^{\beta}\left(\nu^{\alpha}(\mathrm{d} \xi)_{\alpha \beta}-2 \xi_{\alpha} \nabla_{\beta} \nu^{\alpha}\right) \\
& =2 K \bar{\xi}^{\bar{\alpha}}+\bar{g}^{\bar{\alpha} \bar{\beta}}\left(J_{\bar{\beta}}^{\beta} \nu^{\alpha}(\mathrm{d} \xi)_{\alpha \beta}-2 \bar{\xi}^{\bar{\mu}} K_{\bar{\mu} \bar{\beta}}\right) \\
& =-2\left(K^{\bar{\alpha} \bar{\mu}}-\bar{g}^{\bar{\mu} \bar{\alpha}} K\right) \bar{\xi}_{\bar{\mu}}+\bar{g}^{\bar{\alpha} \bar{\beta}} J_{\bar{\beta}}^{\beta}\left(l_{\vec{\nu}} \mathrm{d} \xi\right)_{\beta} \\
& =-2\left(l_{\bar{\xi}} \tilde{b}\right)^{\bar{\alpha}}+\left(J^{*} l_{\vec{\nu}} \mathrm{d} \xi\right)^{\bar{\alpha}}
\end{aligned}
$$

where $\tilde{b}:=b / \operatorname{vol}_{\bar{g}}$. Thus, using that for 2-forms the equality $\delta \star_{g}=\star_{g} \mathrm{~d}$ holds, we can write

$$
\begin{aligned}
\left(J_{\xi}^{(m)}, \bar{J}_{\xi}^{(m)}\right) & =\left(\star_{g} \delta \mathrm{d} \xi+2 \star_{g} l_{\vec{\xi}} \tilde{E},-2 \star_{\bar{g}} l_{\vec{\xi}} \tilde{b}+J^{*} \# l_{\vec{\nu}} \mathrm{d} \xi\right) \\
& =2 \star_{g} l(\tilde{E}, \tilde{b})+\left(\mathrm{d} \star_{g} \mathrm{~d} \xi, J^{*}\left(\nu \wedge \# \mathrm{~d} \xi+\# l_{\vec{\nu}} \mathrm{d} \xi\right)\right)=2 \star_{g} \underline{l}(\tilde{E}, \tilde{b})+\underline{\mathrm{d}}\left(\star_{g} \mathrm{~d} \xi, 0\right)
\end{aligned}
$$


where in the first line we have used the operator $\# \alpha=\star_{g}(\nu \wedge \alpha)$ which satisfies

$\star_{g} \alpha=\nu \wedge \# \alpha+\#_{l_{\vec{\nu}}} \alpha \quad$ and $\quad J^{*} \#=\star_{\bar{g}} J^{*}$

\section{Some tetrad computations}

\section{a. Variations}

$$
\begin{aligned}
\mathbb{d} L^{(t)}= & \frac{1}{2} \mathbb{d T r}_{\eta}\left(\star_{\eta}\{e \wedge e\} \wedge\left(F-\frac{\Lambda}{12}\{e \wedge e\}\right)\right) \\
= & \frac{1}{2} \operatorname{Tr}_{\eta}\left(2 \star_{\eta}\{\mathbb{d} e \wedge e\} \wedge F+\star_{\eta}\{e \wedge e\} \wedge \mathbb{d} F-\frac{\Lambda}{3} \star_{\eta}\{\mathbb{d} e \wedge e\} \wedge\{e \wedge e\}\right) \\
= & \frac{1}{2} \operatorname{Tr}_{\eta}\left(2 \star_{\eta} F \wedge\{e \wedge \mathbb{d} e\}+\star_{\eta}\{e \wedge e\} \wedge \mathcal{D} \mathbb{d} \omega-\frac{\Lambda}{3} \star_{\eta}\{e \wedge e\} \wedge\{e \wedge \mathbb{d} e\}\right) \stackrel{(\mathrm{IV} .3)}{=} \\
= & \operatorname{Tr}_{\eta}\left(-\left[\star_{\eta} F(\Lambda) \wedge e\right] \wedge \mathbb{d e}-\frac{1}{2} \star_{\eta} \mathcal{D}\{e \wedge e\} \wedge \mathbb{d} \omega\right)+\mathrm{dTr}_{\eta}\left(\frac{1}{2} \star_{\eta}\{e \wedge e\} \wedge \mathbb{d} \omega\right), \\
\mathbb{d} \bar{e}^{(t)}-J^{*} \Theta^{(t)}= & -\frac{1}{2} \mathbb{d} \operatorname{Tr}_{\eta}\left((\{N \wedge \mathrm{d} N\}-\bar{\omega}) \wedge \star_{\eta}\{\bar{e} \wedge \bar{e}\}\right)-J^{*} \operatorname{Tr}_{\eta}\left(\frac{1}{2} \star_{\eta}\{e \wedge e\} \wedge \mathbb{d} \omega\right) \\
= & -\frac{1}{2} \operatorname{Tr}_{\eta}\left((\{\mathbb{d} N \wedge \mathrm{d} N\}+\{N \wedge \mathrm{d} \mathbb{d} N\}) \wedge \star_{\eta}\{\bar{e} \wedge \bar{e}\}+2(\{N \wedge \mathrm{d} N\}-\bar{\omega}) \wedge \star_{\eta}\{\mathbb{d} \bar{e} \wedge \bar{e}\}\right) \\
= & -\frac{1}{2} \operatorname{Tr}_{\eta}\left(2\{\mathbb{d} N \wedge \mathrm{d} N\} \wedge \star_{\eta}\{\bar{e} \wedge \bar{e}\}+\mathrm{d}\left(\{N \wedge \mathbb{d} N\} \wedge \star_{\eta}\{\bar{e} \wedge \bar{e}\}\right)-2\{N \wedge \mathbb{d} N\} \wedge \star_{\eta}\{\mathrm{d} \bar{e} \wedge \bar{e}\}\right. \\
& \left.+2 \star_{\eta}(\{N \wedge \mathrm{d} N\}-\bar{\omega}) \wedge\{\mathbb{d} \bar{e} \wedge \bar{e}\}\right) \\
= & -\operatorname{Tr}_{\eta}\left(\{\mathbb{d} N \wedge \mathrm{d} N\} \wedge \star_{\eta}\{\bar{e} \wedge \bar{e}\}-\{N \wedge \mathbb{d} N\} \wedge \star_{\eta}\{\mathrm{d} \bar{e} \wedge \bar{e}\}-\left[\star_{\eta}(\{N \wedge \mathrm{d} N\}-\bar{\omega}) \wedge \bar{e}\right] \wedge \mathbb{d} \bar{e}\right) \\
& -\operatorname{dir}_{\eta}\left(\frac{1}{2}\{N \wedge \mathbb{d} N\} \wedge \star_{\eta}\{\bar{e} \wedge \bar{e}\}\right) .
\end{aligned}
$$

Let us prove that the first term vanishes. First notice that $\varepsilon^{[J K L} N^{M]} \in \Omega_{5}^{0}(M)=\{0\}$. Thus

$$
\begin{aligned}
0 & =5 \varepsilon^{[I J K L} N^{M]} \bar{e}_{K} \wedge \bar{e}_{L} \wedge \mathrm{d} N_{I} \wedge\left(l_{\bar{E}_{J}} \mathbb{d} \bar{e}_{M}\right) \\
& =\left(\varepsilon^{I J K L} N^{M}+\varepsilon^{J K L M} N^{I}+\varepsilon^{K L M I} N^{J}+2 \varepsilon^{L M I J} N^{K}\right) \bar{e}_{K} \wedge \bar{e}_{L} \wedge \mathrm{d} N_{I} \wedge\left(l_{\bar{E}_{J}} \mathbb{\mathbb { e }} \bar{e}_{M}\right) \\
& =-\varepsilon^{I J K L} \bar{e}_{K} \wedge \bar{e}_{L} \wedge \mathrm{d} N_{I} \wedge \mathbb{d} N_{J}=-\star_{\eta}\{\bar{e} \wedge \bar{e}\}_{I J} \wedge \mathrm{d} N^{I} \wedge \mathbb{d} N^{J}
\end{aligned}
$$

where we have used $\mathbb{d} N_{I}=-N_{J} \bar{E}_{I} \mathbb{d} \bar{e}^{J}, N^{I} \bar{E}_{I}^{\bar{\alpha}}=0, N^{I} \mathrm{~d} N_{I}=0$, and $N^{I} \bar{e}_{I}=0$. Let us now rework the second term to obtain the desired expression of Sec. IV D

$$
\begin{aligned}
& \operatorname{Tr}_{\eta}\left(\{N \wedge \mathbb{d}\} \wedge \star_{\eta}\{\mathrm{d} \bar{e} \wedge \bar{e}\}\right)=\{N \wedge \mathbb{d}\}^{I J} \frac{1}{2} \varepsilon_{I J K L}\{\mathrm{~d} \bar{e} \wedge \bar{e}\}^{K L} \\
& \quad=2 N^{I} \mathbb{d} N^{J} \varepsilon_{I J K L}\left(\mathrm{~d} \bar{e}^{K}\right) \wedge \bar{e}^{L}=-2 N^{I} N_{R}\left(l_{\bar{E}}^{J} \mathbb{d} \bar{e}^{R}\right) \varepsilon_{I J K L}\left(\mathrm{~d} \bar{e}^{K}\right) \wedge \bar{e}^{L} \\
& \quad=-2 N^{I} N_{R} \varepsilon_{I J K L}\left(l_{\bar{E}^{J}}\left(\mathbb{d} \bar{e}^{R} \wedge \mathrm{d} \bar{e}^{K} \wedge \bar{e}^{L}\right)+\mathbb{d} \bar{e}^{R} \wedge\left(l_{\bar{E}^{J}} \mathrm{~d} \bar{e}^{K}\right) \wedge \bar{e}^{L}+\mathbb{d} \bar{e}^{R} \wedge \mathrm{d} \bar{e}^{K} \wedge \gamma^{J L}\right) \\
& \quad=-2 N^{I} N_{R} \varepsilon_{I J K L}\left(0+\mathbb{d} \bar{e}^{R} \wedge\left(l_{\bar{E}^{J}} \mathrm{~d} \bar{e}^{K}\right) \wedge \bar{e}^{L}+0\right)=-2 N^{I} N_{R} \varepsilon_{I J K L}\left(l_{\bar{E}^{J}} \mathrm{~d} \bar{e}^{K}\right) \wedge \bar{e}^{L} \wedge \mathbb{d} \bar{e}^{R} \\
& \quad=-N_{R} \varepsilon_{I J K L}\left(l_{\bar{E}^{J}} \mathrm{~d} \bar{e}^{K}\right)\{N \wedge \bar{e}\}^{I L} \wedge \mathbb{d} \bar{e}^{R}=-2 N_{R}\left(l_{\bar{E}^{J}} \mathrm{~d} \bar{e}^{K}\right) \star_{\eta}\{N \wedge \bar{e}\}_{J K} \wedge \mathbb{d} \bar{e}^{R}
\end{aligned}
$$


[1] R. M. Wald, Black hole entropy is the Noether charge, Phys. Rev. D 48, R3427(R) (1993).

[2] R. M. Wald, The thermodynamics of black holes, Living Rev. Relativity 4, 6 (2001).

[3] R. Penrose, Asymptotic Properties of Fields and SpaceTimes, Phys. Rev. Lett. 10, 66 (1963).

[4] R. Penrose, Zero rest-mass fields including gravitation: Asymptotic behaviour, Proc. R. Soc. A 284, 159 (1965).

[5] A. Ashtekar and M. Streubel, Symplectic geometry of radiative modes and conserved quantities at null infinity, Proc. R. Soc. A 376, 585 (1981).

[6] A. Ashtekar, M. Campiglia, and A. Laddha, Null infinity, the BMS group and infrared issues, Gen. Relativ. Gravit. 50, 140 (2018).

[7] A. Ashtekar and B. Krishnan, Isolated and dynamical horizons and their applications, Living Rev. Relativity 7, 10 (2004).

[8] A. Ashtekar, S. Fairhurst, and B. Krishnan, Isolated horizons: Hamiltonian evolution and the first law, Phys. Rev. D 62, 104025 (2000).

[9] A. Ashtekar, J. Engle, and D. Sloan, Asymptotics and Hamiltonians in a first order formalism, Classical Quant. Grav. 25, 095020 (2008).

[10] J. Engle, K. Noui, A. Perez, and D. Pranzetti, Black hole entropy from the $\mathrm{SU}(2)$-invariant formulation of type I isolated horizons, Phys. Rev. D 82, 044050 (2010).

[11] J. Engle, K. Noui, A. Perez, and D. Pranzetti, The SU(2) black hole entropy revisited, J. High Energy Phys. 05 (2011) 016.

[12] V. Iyer and Robert M. Wald, Some properties of the Noether charge and a proposal for dynamical black hole entropy, Phys. Rev. D 50, 846 (1994).

[13] A. Corichi and J. D. Reyes, The gravitational Hamiltonian, first order action, Poincaré charges and surface terms, Classical Quant. Grav. 32, 195024 (2015).

[14] B. P. Dolan, A tale of two derivatives: Phase space symmetries and Noether charges in diffeomorphism invariant theories, Phys. Rev. D 98, 044009 (2018).

[15] J. Margalef-Bentabol and E. J.S. Villaseñor, Geometric formulation of the covariant phase space methods with boundaries, Phys. Rev. D 103, 025011 (2021).

[16] A. Ashtekar and M. Varadarajan, Striking property of the gravitational Hamiltonian, Phys. Rev. D 50, 4944 (1994).

[17] A. Ashtekar, Large Quantum Gravity Effects: Unforeseen Limitations of the Classical Theory, Phys. Rev. Lett. 77, 4864 (1996).

[18] J. Fernando Barbero, G. Iñaki Garay, and E. J. S. Villaseñor, Exact Quantization of Einstein-Rosen Waves Coupled to Massless Scalar Matter, Phys. Rev. Lett. 95, 051301 (2005).
[19] J. Fernando Barbero G and E. J. S. Villaseñor, Quantization of midisuperspace models, Living Rev. Relativity 13, 6 (2010).

[20] T. Jacobson and A. Mohd, Black hole entropy and Lorentzdiffeomorphism Noether charge, Phys. Rev. D 92, 124010 (2015).

[21] R. Oliveri and S. Speziale, Boundary effects in general relativity with tetrad variables, Gen. Relativ. Gravit. 52, 83 (2020).

[22] E. De Paoli and S. Speziale, A gauge-invariant symplectic potential for tetrad general relativity, J. High Energy Phys. 07 (2018) 040.

[23] L. Freidel, M. Geiller, and D. Pranzetti, Edge modes of gravity. Part I. Corner potentials and charges, J. High Energy Phys. 11 (2020) 026.

[24] R. M. Wald, General Relativity (University of Chicago Press, Chicago, 1984).

[25] C. Krishnan and A. Raju, A neumann boundary term for gravity, Mod. Phys. Lett. A 32, 1750077 (2017).

[26] A. Ashtekar and A. Magnon-Ashtekar, On the symplectic structure of general relativity, Commun. Math. Phys. 86, 55 (1982).

[27] J. Frauendiener and G. A. J. Sparling, On the symplectic formalism for general relativity, Proc. R. Soc. A 436, 141 (1992).

[28] A. Ashtekar, L. Bombelli, and O. Reula, The covariant phase space of asymptotically flat gravitational fields, Mechanics, Analysis and Geometry: 200 Years After Lagrange (Elsevier, Amsterdam, 1991), pp. 417-450.

[29] D. Harlow and J.-Q. Wu, Covariant phase space with boundaries, J. High Energy Phys. 10 (2020) 146.

[30] R. Penrose and W. Rindler, Spinors and Space-Time (Cambridge Monographs on Mathematical Physics) (Cambridge University Press, Cambridge, England, 1984).

[31] A. Ashtekar, G. T. Horowitz, and A. Magnon-Ashtekar, A generalization of tensor calculus and its applications to physics, Gen. Relativ. Gravit. 14, 411 (1982).

[32] J. D. Romano, Geometrodynamics vs connection dynamics, Gen. Relativ. Gravit. 25, 759 (1993).

[33] M. Tecchiolli, On the mathematics of coframe formalism and Einstein-Cartan theory-A brief review, Universe 5, 206 (2019).

[34] R. Geroch, Spinor structure of space-times in general relativity. I, J. Math. Phys. (N.Y.) 9, 1739 (1968).

[35] N. Bodendorfer and Y. Neiman, Imaginary action, spinfoam asymptotics and the "transplanckian" regime of loop quantum gravity, Classical Quant. Grav. 30, 195018 (2013).

[36] A. Corichi, I. Rubalcava-Garcia, and T. Vukasinac, Actions, topological terms and boundaries in first order gravity: A review, Int. J. Mod. Phys. D 25, 1630011 (2016). 